\title{
COMPLETENESS FOR COUNTER-DOXA CONDITIONALS - USING RANKING SEMANTICS
}

\author{
ERIC RAIDL \\ Department of Philosophy, University of Konstanz
}

\begin{abstract}
Standard conditionals $\varphi>\psi$, by which I roughly mean variably strict conditionals $\grave{a}$ la Stalnaker and Lewis, are trivially true for impossible antecedents. This article investigates three modifications in a doxastic setting. For the neutral conditional, all impossible-antecedent conditionals are false, for the doxastic conditional they are only true if the consequent is absolutely necessary, and for the metaphysical conditional only if the consequent is 'model-implied' by the antecedent. I motivate these conditionals logically, and also doxastically by properties of conditional belief and belief revision. For this I show that the Lewisian hierarchy of conditional logics can be reproduced within ranking semantics, provided we slightly stretch the notion of a ranking function. Given this, acceptance of a conditional can be interpreted as a conditional belief. The epistemic and the neutral conditional deviate from Lewis' weakest system $\mathbf{V}$, in that ID $(\varphi>$ $\varphi)$ or even $\mathrm{CN}(\varphi>\mathrm{T})$ are dropped, and new axioms appear. The logic of the metaphysical conditional is completely axiomatised by $\mathrm{V}$ to which we add the known Kripke axioms $\mathrm{T} 5$ for the outer modality. Related completeness results for variations of the ranking semantics are obtained as corollaries.
\end{abstract}

\$1. Introduction. In standard conditional logics of the Lewis/ Stalnaker type, a conditional $\varphi>\psi$ is trivially true if $\varphi$ is inaccessible ${ }^{1}$ or (equivalently) impossible with respect to the outer modality of $>$, defined as $\square \theta \equiv \neg \theta>\perp$. Thereby impossibleantecedent conditionals ${ }^{2}$ (i.e., $\varphi>\psi$ such that $\varphi>\perp$ ) create conditional confusionevery consequent is as fine as any other.

In the ontic setting, vacuism (cf. Berto, French, Priest, \& Ripley (2018)) is the thesis that counter-possibles are trivially or vacuously true, as defended by Williamson (2007, 2018). Nonvacuism is the thesis that some counter-possibles are true and some are false (Berto et al., 2018). A usual approach to nonvacuism is to use impossible worlds (cf. Mares \& Fuhrmann (1995), Nolan (1997), Brogaard \& Salerno (2013), Bjerring (2014)). This article investigates nonvacuism in a doxastic setting, where impossibility is doxastic impossibility. As opposed to usual ontic nonvacuist positions, the present analysis does not make use of logically impossible worlds, but uses doxastically impossible worlds instead. Yet, doxastically impossible worlds behave truth-functionally as possible worlds, they are just judged crazy by the doxastic agent.

The article focuses on three modifications of standard conditionals. Impossible-antecedent conditionals, instead of being always true (standard conditional), could be always

Received: September 17, 2017.

2010 Mathematics Subject Classification: 03A05, 03B45, 03B42, 03B47, 03B65, 68T37, 91F20.

Key words and phrases: conditionals, conditionalisation, ranking theory, ranking semantics, counter-possibles, vacuism, nonvacuism.

1 Short for there is no accessible $\varphi$-world.

2 Called "counterpossible" conditionals in the ontic setting. 
false (neutral conditional), ${ }^{3}$ or true or false depending on whether the consequent is absolutely necessary (doxastic conditional), or depending on whether the consequent necessarily follows from the antecedent (metaphysical conditional) with respect to a universal necessity. I motivate these modifications by a conditional-belief interpretation of $>$. Whereas the standard conditional results in believing everything given an impossible proposition, the neutral conditional results in believing nothing, the doxastic conditional in believing only what is absolutely necessary and the metaphysical conditional in believing only what follows universally from the antecedent.

The conditional-belief interpretation of $>$ is obtained by defining $>$ with respect to ranking conditionalisation, the most promising version of conditional belief ${ }^{4}$ (Spohn, 1988, 2012). The implementation of this in a possible world semantics is what I call ranking semantics, an idea introduced by Friedman \& Halpern (2001) within their plausibility semantics (cf. Halpern (2003)). It has been philosophically discussed and motivated by Huber $(2014,2015$, 2017). Spohn (2015) develops his own version and Lauer (2017) uses it to analyse graded belief as a graded modality. Friedman \& Halpern (2001) prove the completeness of VP (i.e., Lewis' $V$ augmented by the axiom P: $\neg(T>\perp)$ ) for the ranking semantics. Raidl (2018) proves completeness results for the outer modalities $\left(\square_{n} \theta \equiv \neg \theta>_{n} \perp\right.$ ) and inner modalities $\left(\bullet_{n} \theta \equiv \top>_{n} \theta\right)$ arising from graded or strength-indexed conditionals $\varphi>_{n} \psi$ corresponding to strength-indexed ranking-theoretic conditional belief.

To show that standard Lewisian conditionals can generally be interpreted in terms of conditional belief, I extend Friedman and Halpern's result and prove that Lewis' hierarchy of conditional logics can be reproduced by slightly stretching the notion of a ranking function. The new truth clauses for impossible-antecedent conditionals can then be related to modified ranking conditionalisation, suggested by Huber (2007a,b, 2014), which I motivate by properties of the induced belief revision, in the spirit of Alchourrón, Gärdenfors, \& Makinson (1985) and Darwiche \& Pearl (1997). Completeness results are proven for the new conditionals. Thereby the article relates ranking semantics to Lewis' logics of ontic conditionals and also attempts a formal unification of the different existing versions of ranking semantics.

The plan of the article is as follows: $\$ 2$ argues for restricting the cases where impossibleantecedent conditionals come out trivially true. $\$ 3$ proves the reproducibility of the Lewisian hierarchy within the generalised ranking semantics and establishes the connection between conditionals and ranking-theoretic conditional belief. $\$ 4$ motivates the modified truth clauses for impossible-antecedent conditionals by properties of conditional belief and belief revision. $\$ 5$ discusses the completeness results for the new conditionals, including also an alternative definition from Huber (2017, footnote 4). $\$ 6$ makes some concluding remarks. Appendix $§ 7$ contains the completeness proofs and additional material.

§2. Impossible-antecedent conditionals. Standard semantics for variably strict conditionals have a truth condition of the following disjunctive form: ${ }^{5}$

- $\varphi>\psi$ is true in world $w$ iff $\psi$ is true in all closest $\varphi$-worlds or $\varphi$ is inaccessible (i.e., no $\varphi$-world is accessible from $w$ ).

3 Discussed by Kment (2014)

4 Provided one wants belief to be closed under conjunction and invariant under fine-grainings of the language, a combination which probabilistic belief cannot meet. However, the framework could be transposed to other plausibility measures.

5 The exact form of the disjuncts depends on the semantics. 
By the trivial-truth condition (second disjunct) all conditionals $\varphi>\psi$ are trivially true for inaccessible antecedents $\varphi$; in particular, $\varphi>\perp$ holds. Conversely, $\varphi>\perp$ implies $\varphi>\psi$ for any $\psi$, by the rule of right-monotonicity (RCM). Thus $\varphi>\psi$ is trivially true for impossible antecedents $\varphi$.

One might hold that the trivial truth of impossible-antecedent conditionals is an expected feature in certain contexts. For example, to mock someone, or more generally, to underline the implausibility of the antecedent, often by a consequent judged even more implausible, or conversely to highlight the necessity (or regularity) of a consequent by stating that not even an absurd antecedent can trump it:

(1) If you pass the exam, I am the Queen of England.

(mocking)

(2) If I win the lottery, then $2+2=5$.

(a-improbability)

(3) If aliens invade the earth tomorrow, I will [still] walk to university. (c-regularity)

Or in particular situations:

(4) If [were] $2+2=5$, [then would be] $2+2+1=6 .^{6}$

(partial truth)

In (4) the speaker could indicate that a student, although having arrived at a false result, did something right, namely she correctly computed that $5+1=6$. The wrong result $2+2+1=6$ would be right, if $2+2$ were 5 (provided $5+1$ were still 6 ).

However, one can also reject impossible-antecedent conditionals. First, analysing the effects in (1)-(4) in terms of an impossible antecedent is not mandatory-it suffices that the antecedent is judged highly implausible. Second, if the above contextual intention is absent (mocking, underlining improbability or regularity or partial truth), one would rather reject impossible-antecedent conditionals. In (1), a speaker without intentions of mocking would manifest crazy beliefs concerning the relation between herself being the Queen of England and her friend passing the exam. Similarly in (2). Removing 'still' in (3), the speaker expresses an irrational decision attitude, since it would be much better to hide. Taken seriously, the speaker in (4) just says $\perp>\perp$, which is trivially valid, if we accept the axiom ID $(\varphi>\varphi)$, but which we may want to reject if we want to avoid $\perp>\psi$ for any $\psi$ in presence of RCM.

Third, whether we should accept certain variations of the above seems less clear:

(5) $\sharp$ If you pass the exam, you don't pass the exam.

(6) $\sharp$ If I win the lottery, you will pass the exam.

(7) $\sharp$ If the sun explodes tomorrow, we will have an exam tomorrow.

(8) $\sharp$ If [were] $2+2=5$, [then would be] $2+2=4$.

(9) $\sharp$ If [were] $2+2=5$, [then would be] $5-2=1$.

Yet, if acceptance of (1)-(4) were due to the trivial-truth condition, then

- the speaker in (1) must accept (5), which has no mocking effect;

- the speaker in (2) must also accept (6), which does not underline any antecedentimprobability;

- the speaker in (3) must also accept (7), which does not underline any consequentregularity;

${ }^{6}$ One needs to treat arithmetic statements as nonanalysable propositional variables or introduce contradictory worlds. 
- $\quad$ and the speaker in (4) must also accept (8) and (9), which do not recover any partial truth.

For these reasons, restricting trivial truth of impossible-antecedent conditionals seems not as unreasonable as standard conditional logics suggest. Later on, I will justify this, based on the connection between the conditional and conditional belief. To establish that connection, I now turn to ranking semantics for conditionals.

§3. Ranking semantics. This section introduces the implementation of ranking theory in ranking semantics and proves the reproducibility of Lewis' hierarchy of conditional logics.

The idea of ranking theory is informally as follows: Propositions are subsets of a nonempty set of worlds $W$. Each world $w \in W$ gets assigned a rank $k(w)$ which is either a natural number or infinity, such that at least one world has rank 0 . Positive numbers represent disbelief of increasing strength, 0 represents absence of disbelief (which is not equivalent to full belief!) and $\infty$ represents maximal disbelief. The rank $\kappa(A)$ of a proposition $A \subseteq W$ is the minimal rank among $A$-worlds. Believing $A$ means to disbelieve the negation (complement of $A$ ). Thus the set of 0 -worlds is the strongest believed proposition. Conditionalising on a (finite) proposition $A$ throws non- $A$-worlds to infinity and uniformly displaces $A$-worlds such that the minimum sits at zero again. Conditional belief given $A$ is belief after conditionalising by $A$.

In what follows, $W$ is a nonempty set of worlds, $\wp(W)$ is the powerset algebra over $W$ and $\mathbb{N}_{\infty}=\mathbb{N} \cup\{\infty\}$ are the extended natural numbers. ${ }^{7}$

Definition 3.1. A ranking mass over $W$ is a function $k: W \longrightarrow \mathbb{N}_{\infty}$ with $k^{-1}[0] \neq \emptyset$.

DEFINITION 3.2. $\kappa$ is a complete negative ranking function (n.r.f.) over $\wp(W)$ iff it is a function $\kappa: \wp(W) \longrightarrow \mathbb{N}_{\infty}$ that satisfies: (1) $\kappa(W)=0$, (2) $\kappa(\emptyset)=\infty$ and (3) for all $\mathcal{S} \subseteq \wp(W): \kappa(\bigcup \mathcal{S})=\min _{A \in \mathcal{S}} \kappa(A)$ (complete minimativity).

Ranking masses and ranking functions are interdefinable. ${ }^{8}$ Thus, writing $\kappa(w)$ instead of $\kappa(\{w\})$ will do no harm. A negative ranking function $\kappa$ is regular iff $\kappa(A)=\infty$ only for $A=\emptyset . \kappa$ represents a grading of disbelief.

DEFINITION 3.3. Let $\kappa$ be a n.r.f. over $\wp(W)$. For $A \in \wp(W)$ such that $\kappa(A)<\infty$, the conditionalisation $\kappa_{A}$ of $\kappa$ by $A$ is defined as follows: for all $B \in \wp(W)$,

$$
\kappa_{A}(B):=\kappa(B \cap A)-\kappa(A)
$$

with $\infty-n:=\infty$ for $n \in \mathbb{N}$.

Definition 3.4. The belief set corresponding to $\kappa$ is $\operatorname{Bel}(\kappa):=\{A \in \wp(W): \kappa(\bar{A})>0\}$. If $\kappa(A)<\infty$, the conditional belief set given $A$, is $\operatorname{Bel}\left(\kappa_{A}\right)$, otherwise conditional belief is undefined in Spohn's account.

$\operatorname{Bel}(\kappa)$ is a proper principal filter, generated by the belief core $[0]_{\kappa}:=\{w \in W: \kappa(\{w\})=$ $0\}$ as $\operatorname{Bel}(\kappa)=\left\{A \in \wp(W):[0]_{\kappa} \subseteq A\right\}$. Analogously for $\operatorname{Bel}\left(\kappa_{A}\right)$, provided $\kappa(A)<\infty$. If defined, conditional belief in $B$ given $A$, i.e., $B \in \operatorname{Bel}\left(\kappa_{A}\right)$, can be interpreted as the acceptance of a Stalnaker-type conditional, yielding $A>B$ iff the closest $A$-worlds are

7 Cf. Spohn (2012, chap. 5) for the definitions.

8 Setting $\kappa_{k}(A):=\min _{w \in A} k(w)$ if $A \neq \emptyset$, and $\kappa_{k}(\emptyset):=\infty$. Conversely: $k_{\kappa}(w)=\kappa(\{w\})$. 
$B$-worlds, or as a Lewis-type conditional, yielding $A>B$ iff there is an $(A \cap B)$-world $w$ such that no $(A \cap \bar{B})$-world is at least as close as $w$.

Let me transform the latter allusion to conditional logic into concrete definitions. The conditional language is $\mathcal{L}_{>}=\mathcal{L} \cup\{>\}$, where $\mathcal{L}=\operatorname{Var} \cup\{\neg, \wedge, \vee, \rightarrow\} \cup\{),(\}$ and Var is a set of propositional variables. The set of sentences $\mathrm{S}\left(\mathcal{L}_{>}\right)$is defined inductively as usual.

DEFinition 3.5. $\mathfrak{R}=\left\langle W,\left(\kappa^{w}\right)_{w \in W}, V\right\rangle$ is a ranked model for $\mathcal{L}_{>}$iff $(1) W \neq \emptyset$, (2) each $\kappa^{w}$ is a complete ranking function over $\wp(W),(3) V: \operatorname{Var} \longrightarrow \wp(W)$ is a valuation.

$\mathfrak{R}$ is $(\kappa m p)^{9}$ iff $\left(\kappa_{A}^{w}(\bar{B})>0\right.$ or $\left.\kappa^{w}(A)=\infty\right)$ implies $w \in \bar{A} \cup B$; weakly centered $(\kappa c w)$ iff $\kappa^{w}(w)=0$; strongly centered ( $\left.\kappa c s\right)$ iff $w$ is the only zero-world of $\kappa^{w}$; reflexive $(\kappa t)$ iff $\kappa^{w}(w)<\infty$; symmetric ( $\kappa$ iv) iff $\kappa^{w}(v)<\infty$ implies $\kappa^{v}(w)<\infty$; euclidean $(\kappa v)$ iff $\kappa^{w}(v), \kappa^{w}(u)<\infty$ implies $\kappa^{v}(u)<\infty$; pointed ( taken by at most one world; point-centered iff centered and pointed; it is regular ( $\mathrm{kreg})$ iff all $\kappa^{w}$ are regular; uniform $(\kappa u)$ iff $\kappa^{w}(v)<\infty$ implies $\kappa^{v}=\kappa^{w}$; and global $(\kappa g)$ iff $\kappa^{w}=\kappa^{v}$ for all $w, v$; it is carnapian iff $W$ are exactly all truth-functions $w$ : $\operatorname{Var} \longrightarrow\{0,1\}$ and $w \in V(p)$ iff $w(p)=1$.

$\mathfrak{R}$ is a generalised ranked mode ${ }^{10}$ iff instead of (2), we have $\left(2^{\prime}\right)$ each $\kappa^{w}$ is a complete generalised ranking function over $\wp(W)$, i.e., $k^{w}: W \longrightarrow \mathbb{N}_{\infty}$ is just a function, ${ }^{11}$ and $\kappa^{w}(A)=\kappa_{k}^{w}, \kappa_{A}^{w}$ remain defined as above. ${ }^{12}$

Centering implies weak centering, regular implies reflexive and euclidean, global implies uniform, which implies symmetric and euclidean. Truth for propositional variables and the standard connectives are defined as usual, and the standard conditional is defined by

(1) $w \vDash_{\mathfrak{R}} \varphi>\psi \quad$ iff $\quad \kappa^{w}([\varphi])=\infty$ or $\kappa_{[\varphi]}^{w}([\neg \psi])>0 . \quad$ (standard conditional)

The sets $[\psi]:=[\psi]^{\mathfrak{R}}=\left\{v \in W: v \vDash_{\mathfrak{R}} \psi\right\}$ are co-inductively defined. I abbreviate $\kappa^{w}(\varphi)=\kappa^{w}([\varphi])$ and $\kappa_{\varphi}^{w}=\kappa_{[\varphi]}^{w}$. For $\mathbf{R}$ a class of ranked models, $\varphi \in \mathrm{S}\left(\mathcal{L}_{>}\right)$is valid in $\mathbf{R}$, written $\mathbf{R} \vDash \varphi$, iff for every world $w$ of every model $\mathfrak{R} \in \mathbf{R}, w \vDash_{\mathfrak{R}} \varphi$.

Huber (2014) considers regular ranked models, Spohn (2015) global-carnapian ranked models, and both restrict the language to the fragment with non-nested conditionals. Friedman \& Halpern (2001, Theorem 8.6(c)) prove that VP-i.e., Lewis' V, augmented by the axiom $\mathrm{P},{ }^{13}$ that is $\neg(T>\perp)$-is complete for ranked models. However, they did not develop the correspondence theory for the hierarchy of conditional logics. Spohn (2015) claimed V to be complete for his semantics, and Huber (2014) for the regular ranking semantics, where in each case at least $\mathrm{P}$ is missing. This motivates the completeness proofs in this section. ${ }^{14}$

We consider the following axiomatisation of Lewis' weakest logic $\mathrm{V}$ : $\mathrm{V}$ is the smallest subset of $\mathrm{S}\left(\mathcal{L}_{>}\right)$which is

$9(\kappa \mathrm{x})$ is the ranking semantics version of the property $(\mathrm{x})$ of a selection model (see $\S 7.2)$ which corresponds to conditional axiom $(\mathrm{X})$, with the exception of (reg) which has no corresponding axiom and (cw) which is equivalent to (mp) and has corresponding axiom (MP). (t), (iv), (v) are the properties corresponding to the known Kripke Axioms (T), (4), (5) for the outer modality. For the properties (t), (iv), (v) extended to graded inner modalities and the correspondence theory of these, see Raidl (2018, Definition 3, Corollary 2).

10 Only used in Theorem 3.6.

11 Where possibly $\kappa^{-1}[0]=\emptyset$ !

12 Thus (1) of Definition 3.3 might fail for $\kappa^{w}$, but $\kappa_{A}^{w}$ remains a n.r.f. if $\kappa^{w}(A)<\infty$ !

13 C6 in Friedman \& Halpern (2001), or Lewis' (1973) normality N.

14 For a comparison, see $\$ 7.1$. 
- $\quad$ closed under modus ponens for $\rightarrow(\mathrm{MoPo})$ and the rules

$$
\left.\frac{\varphi \leftrightarrow \varphi^{\prime}}{(\varphi>\chi) \leftrightarrow\left(\varphi^{\prime}>\chi\right)} \quad \text { (RCEA, LLE, RC1 }\right)^{15} \quad \frac{\varphi \rightarrow \varphi^{\prime}}{(\chi>\varphi) \rightarrow\left(\chi>\varphi^{\prime}\right)} \quad \text { (RCM, RW, RC2) }
$$

- and contains all substitution instances of propositional tautologies and all instances of

$$
\begin{aligned}
& \varphi>\varphi \\
& ((\varphi>\psi) \wedge(\varphi>\chi)) \rightarrow(\varphi>(\psi \wedge \chi)) \\
& ((\varphi>\chi) \wedge(\psi>\chi)) \rightarrow((\psi \vee \varphi)>\chi) \\
& ((\varphi>\chi) \wedge(\varphi>\psi)) \rightarrow((\varphi \wedge \psi)>\chi) \\
& ((\varphi>\chi) \wedge \neg(\varphi>\neg \psi)) \rightarrow((\varphi \wedge \psi)>\chi)
\end{aligned}
$$

(CV, RM, C5)

THEOREM 3.6. $\mathrm{V}$ is sound and complete for generalised ranked models. VP $=\mathrm{V}+\mathrm{P}$ is sound and complete for ranked models.

Proof. See p. 880.

$\mathrm{V}$ is equivalent to the axiom system C0 used by Lewis (1971). ${ }^{17}$ The inner modality $(\square \varphi \equiv \top>\varphi)$ represents actual belief and the outer modality $(\square \varphi \equiv \neg \varphi>\perp)^{18}$ represents a background assumption which I will call doxastic necessity. We have $\square \varphi \rightarrow$ $\square \varphi$. P is the probabilistic consistency axiom, imposing $\neg \square \perp$, which implies $\neg \square \perp$, and since both necessities act like a Kripke necessity, we obtain the better-known axiom D $(\square \varphi \rightarrow \diamond \varphi)$ for both modalities. ${ }^{19}$ From now on, when not mentioned otherwise, we restrict considerations to ranked models and drop the generalised ranked models.

Extending this result, we obtain

THEOREM 3.7. For ranked models with property $(\kappa x)$, VP augmented by X is sound and complete. $^{20}$

\begin{tabular}{l|lr|r} 
Property $(\kappa x)$ & Scheme & VP $+\mathrm{X}$ & Equivalent \\
\hline reflexive $(\kappa \mathrm{t})$ & $(\varphi>\perp) \rightarrow \neg \varphi$ & $\mathrm{T}$ & $\mathrm{V}+\mathrm{T} \equiv \mathrm{VT}$ \\
symmetric $(\kappa \mathrm{iv})$ & $(\varphi>\perp) \rightarrow(\neg(\varphi>\perp)>\perp)$ & 4 & \\
euclidean $(\kappa \mathrm{v})$ & $\neg(\varphi>\perp) \rightarrow((\varphi>\perp)>\perp)$ & 5 & \\
weakly centered $(\kappa \mathrm{cw}$ or mp) & $(\varphi>\psi) \rightarrow(\varphi \rightarrow \psi)$ & $\mathrm{MP}$ & $\mathrm{V}+\mathrm{MP} \equiv \mathrm{VW}$ \\
strongly centered $(\kappa \mathrm{cs})$ & $\mathrm{MP}+(\varphi \wedge \psi) \rightarrow(\varphi>\psi)$ & $\mathrm{MP}+\mathrm{CS}$ & $\mathrm{VT}+\mathrm{CS} \equiv \mathrm{VC}$ \\
pointed $(\kappa \mathrm{cem})$ & $(\varphi>\psi) \vee(\varphi>\neg \psi)$ & $\mathrm{CEM}$ & \\
point-centered $(\kappa \mathrm{cem}, \mathrm{cw})$ & & $\mathrm{MP}+\mathrm{CEM}$ & $\mathrm{VT}+\mathrm{CEM} \equiv \mathrm{C} 2$ \\
uniform $(\kappa \mathrm{u})$ & $(\varphi>\psi) \rightarrow(\neg(\varphi>\psi)>\perp)$ & $\mathrm{U}$ &
\end{tabular}

15 In (X, Y, Z), X refers to Chellas' notation used here, Y to the KLM-tradition and Z to Friedman and Halpern's notation.

16 (CSO) $((\varphi>\psi) \wedge(\psi>\varphi)) \rightarrow((\varphi>\chi) \leftrightarrow(\psi>\chi))$ is equivalent, given RCEA, RCM, CC, ID, CA (see Lemma 7.6).

17 An earlier version of the article proved equivalence. The reader is now referred to Unterhuber (2016, Theorem 27), where our $\mathrm{V}$ is denoted as the KLM-system $\mathbf{R}$ for the full language and Lewis' $\mathrm{C} 0$ is denoted as $\mathbf{V}$.

18 Equivalent to $\neg \varphi>\varphi$, given ID, CC, RCM.

19 For this reason P was simply called "D" in Raidl (2018).

20 Given $\mathrm{T}$, the axiom $\mathrm{P}$ becomes redundant. 
Proof. See p. 881, Appendix.

P, T, MP, CS, and CEM are ever stronger centering assumptions. MP implies T, which implies P; CS+T implies MP, and CEM+T implies CS and therefore MP. VW and VC are used by Lewis, and C2 is Stalnaker's system. The above result shows that Lewis' hierarchy can be reproduced using ranking functions instead of systems of spheres, ${ }^{21}$ by slightly stretching the notion of a ranking function to allow for generalised ranking functions. ${ }^{22}$ Therefore that hierarchy can be interpreted as a collection of logics for belief-conditionals, i.e., conditionals based on conditional belief. Let me briefly explain the axioms in terms of conditional belief.

$\mathrm{RCM}$ expresses upwards closure of conditional belief, CC closure under conjunction, and $\mathrm{CN}(\varphi>T)$, implied by RCM and ID, expresses that the tautology is conditionally believed. P expresses consistency of actual belief. Let us write $\operatorname{Bel}_{X}^{*}=\operatorname{Bel}\left(\kappa_{X}\right)$. Then RCEA expresses that conditional belief is extensional, i.e., concordant for logically equivalent sentences, ID that the conditionalisation is successful, i.e., after conditionalising on $\varphi, \varphi$ is believed. CMon imposes that conditional belief satisfies cautious monotonicity: if $C$ and $B$ are believed in $\mathrm{Bel}_{A}^{*}$ then $C$ remains believed in $\mathrm{Bel}_{A \cap B}^{*}$. $\mathrm{CV}$ expresses that conditional belief satisfies rational monotonicity: if $C$ is believed in $\mathrm{Bel}_{A}^{*}$ and $B$ is compatible with $\mathrm{Bel}_{A}^{*}$ then $C$ remains believed in $\mathrm{Bel}_{A \cap B}^{*}$. Finally, CA expresses: if $C \in \mathrm{Bel}_{A}^{*}$ and $C \in \mathrm{Bel}_{B}^{*}$ then $C \in \mathrm{Bel}_{A \cup B}^{*}{ }^{23,24}$

Furthermore, CEM says that conditional belief is complete, CS that conjunctive truth implies conditional belief, and MP that conditional belief is at least as strong as the material implication. Reinterpreting these for the inner modality, we would obtain that actual belief is true (MP), complete (CEM) or implied by truth (CS), respectively. Therefore, MP, CS, and CEM only make sense if actual belief is knowledge-like. T says that doxastic necessity is factual, 4 that it is positively and 5 that it is negatively introspectible. $U$ means that the agent knows (in the sense of $\square$ ) her conditional beliefs. Axioms 4, 5 and $U$ are the only axioms involving a nested conditional.

COROLLARY 3.8. VT5 is sound and complete for regular ranked models, and VPU for global (and even global-carnapian) ranked models.

Proof. See p. 881 in the Appendix.

In the non-nested fragment used by Huber and Spohn, the regular ranking semantics is indistinguishable from the reflexive ranking semantics (VT) and the global ranking semantics is indistinguishable from the ranking semantics simpliciter (VP). ${ }^{25}$

21 Reflexivity is Lewis' total reflexivity, globality Lewis' local absoluteness, (iv) and (v) correspond to Lewis' local uniformity.

22 It would have sufficed to extend ranking functions by the abnormal $\kappa_{\perp}(A)=\infty$ for all $A \in$ $\wp(W)$.

23 Given success, rational monotonicity is equivalent to the AGM postulate of super-expansion, and $\mathrm{CA}$ is equivalent to the AGM postulate of sub-expansion.

24 Instead of using conditionalisation in the truth clause for the conditional, one could have chosen any other revision having these properties, e.g., for fixed $n>0: \kappa_{A}^{n}(w)=\kappa(w)-\kappa(A)$ if $w \in A$, and $\kappa_{A}^{n}(w)=\kappa(w)-\kappa(\bar{A})+n$ if $w \notin A$.

25 Over the non-nested fragment of $\mathrm{S}\left(\mathcal{L}_{>}\right)$we obtain as a consequence: VT is sound and complete for the regular ranking semantics used by Huber (2014) and VP is sound and complete for Spohn's (2015, pp. 10-11) semi-epistemic validity, provided his incomplete truth-condition (1e) is read 
If we proceed to nonstandard belief with threshold $z>0$, defining $\operatorname{Bel}_{\mathcal{K}}^{z}:=\{A \in$ $\wp(W): \kappa(\bar{A})>z\}$ and $w \vDash_{\mathfrak{R}} \varphi>\psi$ iff $\kappa^{w}([\varphi])=\infty$ or $\kappa_{\varphi}^{w}([\neg \psi])>z$, everything remains the same, except that $\mathrm{CV}$ is lost. ${ }^{26}$ Correspondence and completeness results for the outer and inner modalities of these graded or strength indexed conditionals (with $z \in \mathbb{N}$ ) are analysed in Raidl (2018). I conjecture that the complete axiomatisation for the ranking semantics with $z$-belief $(z>0)$ is $B+P+M$, where B is Burgess' (1981) axiomatic system $(\mathrm{V}-\mathrm{CV})$ and $\mathrm{M}$ is

$$
((\varphi \vee \psi)>\chi) \rightarrow((\varphi>\chi) \vee(\psi>\chi)) .
$$

This casts doubt on the interpretation freedom, suggested by Matthias Hild (cf. Spohn $(2012,76-77))$, and is a counterexample to the claim of Spohn $(2015,9)$ that shifting the belief threshold from 0 to $z>0$ would not essentially change the theory: CV-violation proves it does.

The point was to show that, in the framework of ranking semantics, conditionals can be interpreted as corresponding to conditional beliefs and the Lewisian hierarchy of (standard) conditional logics can be reproduced, provided we slightly stretch the notion of a ranking function. Based on this, I will now investigate impossible-antecedent conditionals as counter-doxa conditionals, meaning that the antecedent is impossible with respect to the outer doxastic modality. I will justify different modifications of the standard conditional by properties of conditional belief.

§4. Counter-doxa conditionals. This section introduces and justifies the new conditionals by semantic and doxastic considerations. ${ }^{28}$

In the standard ranking semantics, the conditional $\varphi>\psi$ is true in $w$ iff in $w$ either $\varphi$ is doxastically impossible $\left(\kappa^{w}(\varphi)=\infty\right)$ or $\psi$ is believed conditional on $\varphi$. In the exceptional case (i.e., if $\varphi$ is doxastically impossible), the conditional could be either undefined, or uniformly true (standard conditional), or uniformly false (neutral conditional), or true or false depending on whether the consequent is absolutely necessary, i.e., $[\psi]^{\mathfrak{R}}=W$ (doxastic conditional), or true or false depending on whether the consequent is implied by the antecedent (metaphysical conditional). The latter three yield the following new truth clauses (replacing clause (1) on p. 865):

(2) $w \vDash_{\mathfrak{R}} \varphi \triangleright \psi \quad$ iff $\quad \kappa_{\varphi}^{w}(\neg \psi)>0 \& \kappa^{w}(\varphi)<\infty$.

(neutral)

(3) $w \vDash_{\mathfrak{R}} \varphi \triangleright \psi$ iff

$$
\left(\kappa_{\varphi}^{w}(\neg \psi)>0 \& \kappa^{w}(\varphi)<\infty\right) \text { or }\left([\psi]=W \& \kappa^{w}(\varphi)=\infty\right) .
$$

(doxastic)

(4) $w \models_{\mathfrak{R}} \varphi \triangleright \psi \quad$ iff

$$
\left(\kappa_{\varphi}^{w}(\neg \psi)>0 \& \kappa^{w}(\varphi)<\infty\right) \text { or }\left([\varphi] \subseteq[\psi] \& \kappa^{w}(\varphi)=\infty\right) . \quad \text { (metaphysical) }
$$

$\checkmark$ implies $\triangleright$, which implies $\triangleright$, which implies the standard conditional $>.{ }^{29}$

as (1e.1) (see the Appendix, p. 877 for these). If regularity is imposed instead, it would be VT. Furthermore, VW (not VC) is sound and complete for Spohn's epistemic validity $\models^{e}$.

26 Consider $W=\left\{w_{0}, w_{1}, w_{2}, w_{3}\right\}$ and threshold $z=1$. Consider $A=\left\{w_{1}, w_{2}, w_{3}\right\}, B=$ $\left\{w_{2}, w_{3}\right\}$ and $C=\left\{w_{1}, w_{2}\right\}$, where the indices are the ranks of the worlds. For $\kappa_{A}, C$ is believed and $B$ is possible ( $\bar{B}$ is not believed). But in $\kappa_{A \cap B}, C$ is not believed any more.

$27 \mathrm{M}$ is weaker than CV. M was discussed by Loewer (1979) and Mayer (1981).

28 Similar modifications could be given in a Lewis-, Chellas-, or Segeberger-semantics.

29 Generally, a neutral conditional is definable from a standard conditional by $\varphi>\psi \equiv(\varphi>\psi) \wedge$ $\neg(\varphi>\perp)$, and in the presence of an absolute-necessity $\boxminus$, defined by a universal relation $R=$ 
The neutral conditional rejects all impossible-antecedent conditionals. The doxastic and the metaphysical conditional accept only some impossible-antecedent conditionals. A doxastic impossible-antecedent conditional can only be true for a consequent reflecting a strong background assumption (here model-truth). This is suitable for the cases of 'underlining the regularity or necessity of the consequent' (cf. §2). A metaphysical impossibleantecedent conditional is only true for consequents model-implied by the antecedent (i.e., $\left.[\varphi]^{\mathfrak{R}} \subseteq[\psi]^{\mathfrak{R}}\right)$. Model implication is weaker than logical implication. The metaphysical conditional thus preserves (ID) $\varphi>\varphi$ as well as the idea that a conditional is a kind of implication. Since the semantics has no way to differentiate between grades of doxastic impossibility, ${ }^{30}$ the only option is closure under model-implication (RCM). The metaphysical conditional is then obtained by imposing ID and RCM. In fact, the metaphysical conditional is the smallest RCM-extension of the doxastic conditional that validates ID.

A modified conditionalisation, defined in the case $\kappa(A)=\infty$, would allow adopting the generalised truth condition

(0) $\quad w \vDash_{\mathfrak{R}} \varphi>\psi \quad$ iff $\quad \kappa_{\varphi}^{w}(\neg \psi)>0$.

(conditional belief)

Spohn (2012, Definition 5.15) bans conditionalisation on impossible propositions, presumably for philosophical reasons. A rational agent should never remove a maximal (infinite) doubt. This is unsatisfactory in two respects. From a philosophical point of view, there are situations where maximal disbelief actually was removed (e.g., the doubt of speed nonadditivity or the noneuclideanity of physical space) and if maximal disbelief is not equivalent with logical impossibility, it should be possible to remove such disbeliefs if disbelief is fallible. From a formal point of view, all concepts defined in terms of conditionalisation are undefined in the exceptional case; in particular, we had to invoke an exceptional definition for the conditional.

There are several options for the exception, besides the option of undefinedness:

Definition 4.1. For $\kappa(A)=\infty$ and $\bar{B} \in \wp(W),{ }^{31}$ exceptional conditionalisation is called.... ${ }^{32}$

abnormal iff $\kappa_{A}(\bar{B})=\infty$, doxastic iff $\kappa_{A}(\bar{B})= \begin{cases}\infty & \text { if } B=W, \\ 0 & \text { otherwise. }\end{cases}$

neutral iff $\kappa_{A}(\bar{B})=0$, metaphysical iff $\kappa_{A}(\bar{B})= \begin{cases}\infty & \text { if } A \subseteq B, \\ 0 & \text { otherwise }\end{cases}$

Call the resulting conditionalisations each by the same name, respectively. Only the doxastic and metaphysical conditionalisation always yield a new ranking function (except when $A=\emptyset$ for the metaphysical conditionalisation). Using the uniform scheme (0), abnormal, neutral, doxastic and metaphysical conditionalisation produce the truth condition (1), (2), (3) and (4), respectively. The exceptional clauses can then be motivated doxastically, by

$W^{2}$ (or at least implying the outer modality $\square$ of $>$ ), the doxastic conditional is definable by $\varphi \triangleright \psi \equiv(\varphi \triangleright \psi) \vee \boxminus \psi$ and the metaphysical conditional by $\varphi \triangleright \psi \equiv(\varphi \triangleright \psi) \vee \boxminus(\varphi \rightarrow \psi)$.

30 Unless ordinal-valued ranking functions are used.

31 Taking $\bar{B}$ simplifies the relation to the conditional.

32 Goldszmidt \& Pearl (1996, 63) used the abnormal, Huber (2007a, 517) the neutral, Huber (2006, 464) and Huber $(2007 b, 303)$ the doxastic, and Huber (2014, 2185) the metaphysical conditionalisation. 
properties of conditionalisation, conditional belief and belief revision. This perspective yields the following.

The abnormal exception coincides with the inconsistent belief set $K_{\perp}$ in AGM-revision theory (cf. Alchourrón et al., (1985)) as well as with the inconsistent probability $P_{\perp}$ (1 everywhere) in probabilistic revision theory (Gärdenfors, 1982). This is based on the idea that if the agent accepts a doxastically impossible proposition according to her current state, everything becomes believed, even the contradiction.

The neutral exception has the opposite result. Conditionalising on a doxastically impossible proposition, the agent loses all her beliefs, even that in the tautology, and becomes completely belief-abstemious. These two exceptional definitions (together with undefinedness) are break-downs of the belief system and yield a uniform treatment of the exceptional conditional. By contrast, the doxastic and metaphysical conditionalisation result in new ranking functions (if $A \neq \emptyset$ for the latter) and the exceptional value is not uniform.

The doxastic conditionalisation refines the neutral conditionalisation, by setting $\kappa_{A}(\emptyset)=$ $\infty$, ensuring that the result remains a ranking function. Thereby an agent conditionalising on a doxastically impossible proposition resets her belief to consistent neutrality, i.e., believes only the tautology. The agent loses all her beliefs, except for the weakest one.

The metaphysical conditionalisation further refines $\kappa_{A}(\bar{B})=\infty$ if $A \subseteq B$, which ensures that the result remains a ranking function, except for $A=\emptyset$, in which case the result coincides with the abnormal conditionalisation. By this, an agent conditionalising on a doxastically impossible but noncontradictory proposition will believe at least this proposition and everything which follows logically. ${ }^{33}$ The agent resets her belief to the accepted antecedent proposition $A$, which becomes inconsistent only if $A$ is a contradiction.

A dynamical motivation can now be given by considering the induced AGM belief revision Bel $\mapsto \operatorname{Bel} * A$ (Alchourrón et al., 1985), where $\operatorname{Bel}=\operatorname{Bel}(\kappa)$ and $\operatorname{Bel} * A=$ $\operatorname{Bel}\left(\kappa_{A}\right)$. For $X \subseteq \wp(W)$, let $\mathrm{Cl}(X)$ be the filter spanned by $X$ (i.e., the smallest filter containing $X)$. Algebraic AGM expansion is then defined as $\mathrm{Bel}+A=\operatorname{Cl}(\operatorname{Bel} \cup\{A\})$. Rank-conditionalisation does in fact reproduce a kind of AGM belief revision:

THEOREM 4.2. If $\kappa$ and $\kappa^{\prime}$ are regular, conditionalisation satisfies the following algebraic AGM postulates: ${ }^{34}$

(*1) $\operatorname{Bel}\left(\kappa_{A}\right)$ is a quasi-filter (i.e., is closed under $\cap$ and $\subseteq$ ), ${ }^{35}$

(closure)

(*2) $A \in \operatorname{Bel}\left(\kappa_{A}\right)$,

(*3) $\operatorname{Bel}\left(\kappa_{A}\right) \subseteq \operatorname{Bel}(\kappa)+A$,

(inclusion)

(*4) if $\bar{A} \notin \operatorname{Bel}(\kappa)$ then $\operatorname{Bel}(\kappa) \subseteq \operatorname{Bel}\left(\kappa_{A}\right)$,

(preservation)

(*5) if $A \neq \emptyset$ then $\bigcap \operatorname{Bel}\left(\kappa_{A}\right) \neq \emptyset$,

(consistency)

(*6) if $\kappa$ and $\kappa^{\prime}$ are order-isomorphic, then $\operatorname{Bel}\left(\kappa_{A}\right)=\operatorname{Bel}\left(\kappa_{A}^{\prime}\right)$,

(extensionality)

(*7) $\operatorname{Bel}\left(\kappa_{A \cap B}\right) \subseteq \operatorname{Bel}\left(\kappa_{A}\right)+B$,

(sub-expansion)

(*8) if $\bar{B} \notin \operatorname{Bel}\left(\kappa_{A}\right)$, then $\operatorname{Bel}\left(\kappa_{A}\right)+B \subseteq \operatorname{Bel}\left(\kappa_{A \cap B}\right)$.

(super-expansion)

33 This is the most natural extension of conditionalisation. Conditionalisation (where defined) may be characterised as the only categorical rank update (i.e., setting $\bar{A}$ to infinity, by remaining a ranking function) which preserves all distances within $A$. Extending conditionalisation to $\kappa(A)=$ $\infty$ by this characterisation yields the metaphysical conditionalisation.

34 These are algebraic versions of the reformulation by Darwiche \& Pearl (1997). Vacuity (i.e., $\bar{A} \notin \operatorname{Bel}(\kappa)$ implies $\left.\operatorname{Bel}(\kappa)+A=\operatorname{Bel}\left(\kappa_{A}\right)\right)$ was replaced by the weaker preservation.

35 In view of $* 5$ and the latter comparison, this translation is more useful than to ask for $\operatorname{Bel}\left(\kappa_{A}\right)$ to be a filter. 
If $\kappa$ is not regular, $\operatorname{Bel}\left(\kappa_{A}\right)$ is only defined for $\kappa(A)<\infty$ and then the postulates remain valid (provided $\kappa(A \cap B)<\infty$ for $* 7$ and $* 8$ ).

Proof. Standard exercise.

Exceptional conditionalisations extend the induced belief revision to propositions $A$ with $\kappa(A)=\infty$ and therefore generally to all ranking functions (not just regular ones). They do so in different ways:

THEOREM 4.3. The abnormal, the neutral, the doxastic and the metaphysical conditionalisation satisfy *1, *3, *4, *7, *8 over all ranking functions. But they differ with respect to $* 2$ and $* 5$ :

\section{Abnormal conditionalisation satisfies $* 2$ and}

(*5.1) $\bigcap \operatorname{Bel}\left(\kappa_{A}\right) \neq \emptyset$ if $\kappa(A)<\infty$, else $\operatorname{Bel}\left(\kappa_{A}\right)=\wp(W)$. (partially improper) Neutral conditionalisation violates $* 2$, but satisfies

(*5.2) $\bigcap \operatorname{Bel}\left(\kappa_{A}\right) \neq \emptyset$ if $\kappa(A)<\infty$, else $\operatorname{Bel}\left(\kappa_{A}\right)=\emptyset$.

(partially empty)

Doxastic conditionalisation violates $* 2$, but satisfies

(*5.3) $\bigcap \operatorname{Bel}\left(\kappa_{A}\right) \neq \emptyset$ if $\kappa(A)<\infty$, else $\operatorname{Bel}\left(\kappa_{A}\right)=\{W\} . \quad$ (minimally proper)

Metaphysical conditionalisation satisfies $* 2$ and

(*5.4) $\bigcap \operatorname{Bel}\left(\kappa_{A}\right) \neq \emptyset$ if $\kappa(A)<\infty$, else $\operatorname{Bel}\left(\kappa_{A}\right)=\operatorname{Cl}(A) . \quad$ (minimal success)

\section{Proof. Clear.}

Therefore the abnormal and the neutral conditionalisation violate $* 5$, and the neutral conditionalisation does not always produce a belief filter. Exceptional conditionalisations can then be justified as different extensions of conditionalisation to $\kappa(A)=\infty$, preserving certain AGM-axioms. Metaphysical conditionalisation preserves all axioms, including *2 and $* 5$. Abnormal conditionalisation preserves all axioms except $* 5$. Doxastic conditionalisation preserves all axioms except $* 2$. Neutral conditionalisation preserves all axioms, except $* 2$ and $* 5$.

We have thus obtained a certain convergence of the semantic and the doxastic motivation for the four conditionals. The complete axiomatisations for the new conditional logics provide an additional characterisation.

\$5. Completeness. I here provide and discuss the complete axiomatisations of the three new conditional logics $(\$ 5.1-5.3)$ and an alternative truth definition of the conditional (§5.4). The proofs can be found in $\S 7.4$.

\subsection{Neutral ranking semantics.}

THEOREM 5.1. AXn is sound and complete for the neutral ranking semantics, where AXn is V - ID (i.e., MoPo, RCEA, RCM, PT, CC, CA, CMon, ${ }^{36} \mathrm{CV}$ ), augmented by

$$
\begin{aligned}
& \neg(\varphi \triangleright \perp) \\
& (\varphi \triangleright \top) \rightarrow(\varphi \triangleright \varphi)
\end{aligned}
$$

\footnotetext{
36 Now CSO is equivalent to CMon + CA2, given the remaining axioms.

37 Con stands for conditional consistency. Con is weaker than Aristotle's Thesis (AT): $\neg(\varphi>\neg \varphi)$, used in Unterhuber (2016). But here AT holds by RCM + ID1 + CC.
} 


$$
\begin{aligned}
& T \triangleright T \\
& ((\varphi \wedge \psi) \triangleright T) \rightarrow(\varphi \triangleright T) \\
& (\neg(\varphi \triangleright \varphi) \wedge(\psi \triangleright \chi)) \rightarrow((\varphi \vee \psi) \triangleright \chi)
\end{aligned}
$$

Proof. Theorem 7.10 and $7.12 .^{38}$

If one wants to reject the trivial truth of impossible-antecedent conditionals, in particular $\varphi>\perp$ when $\varphi$ is impossible, the radical option is to simply impose $\neg(\varphi>\perp)$. Thus ID $(\varphi>\varphi)$ must fail, since $\perp>\perp$ fails. This suggests removing ID from the axioms, by which we also remove $\mathrm{CN}(\varphi>\top) .{ }^{39}$ The neutral conditional can then be motivated by arguing that ID should fail only for doxastically impossible propositions. ${ }^{40}$ Con entails P. However, Con is stronger since it excludes all contradictory conditionals $\varphi>\perp$. We thereby lose the standard characterisation of impossible antecedents! Let us therefore say that $\varphi$ is doxastically possible (in the neutral ranking semantics) iff the ignorant conditional $\varphi \backslash \top$ holds (this is the new outer possibility $\varphi$ ). Then ID1 restores ID for doxastically possible antecedents, as desired. By RCM, ID1 can be strengthened to the principle that CN and ID are equivalent and thus indistinguishable. Therefore ID holds exactly for doxastically possible propositions. Hence $\varphi \triangleright \top$ and $\varphi \triangleright \varphi$, both express that $\varphi$ is doxastically possible. ID2 then posits that at least $T$ is doxastically possible. CMon2 is a closure requirement on doxastic possibility: any conjunct in a doxastically possible conjunction is also possible. ${ }^{41}$ Dually, doxastic necessity $\varphi=\neg(\neg \varphi>T)$ is upwards closed ${ }^{42}$ and has the remaining proper filter properties, i.e., $\boldsymbol{\square} \top$ (by Con), $\neg \mathbf{\square} \perp$ (equivalent to ID2) and $(\boldsymbol{\square} \varphi \wedge \boldsymbol{\square} \psi) \rightarrow \mathbf{\square}(\varphi \wedge \psi) .{ }^{43}$ For regular ranking functions, $\varphi \triangleright \psi$ deviates from the standard conditional $\varphi>\psi$ only because CN and ID fail when $\varphi \equiv \perp$. Finally, CA2 says that the antecedent in a conditional can be weakened by an impossible disjunct. ${ }^{44}$

In a nutshell: In the neutral ranking semantics all contradictory conditionals $\varphi \triangleright \perp$ are false (Con). $\varphi>T$ expresses that $\varphi$ is doxastically possible and the dual necessity is belief-like (of the Kripke type KD). Only doxastically possible propositions validate ID (ID1, RCM) and at least the tautology $\mathrm{T}$ is doxastically possible (ID2). For doxastically impossible antecedents, all conditionals are false. ${ }^{45}$

\subsection{Doxastic ranking semantics.}

THEOREM 5.2. AXd is sound and complete for the doxastic ranking semantics, where $\mathrm{AXd}$ is $\mathrm{V}$ - ID, augmented by

38 This also yields completeness of AXn in the non-nested fragment for Spohn's (2015) semiepistemic validity, if his incomplete truth condition (1e) is read as (1e.2) (see the Appendix, p. 877 for these).

39 ID implies CN, given RCM.

40 Semantically, $\varphi$ is doxastically impossible for $\kappa^{w}$ iff $\kappa^{w}(\varphi)=\infty$. Logically, $\varphi$ is doxastically impossible iff $\varphi>\perp$.

41 By the $\mathrm{CN}-\mathrm{ID}$ equivalence, $\mathrm{CMon} 2$ is equivalent to $\mathrm{F}$ in Theorem 5.2.

42 Assume $\square \varphi$, i.e., $\neg(\neg \varphi \neg T)$, thus $\neg((\neg \varphi \wedge \neg \chi) \triangleright T)$ (CMon2). Suppose $\varphi$ logically implies $\chi$. Then $\varphi \vee \chi \equiv \chi$ and $(\neg \varphi \wedge \neg \chi) \equiv \neg(\varphi \vee \chi) \equiv \neg \chi$. Thus $\neg(\neg \chi \vee T)$ (RCEA). Hence $\chi$.

43 Suppose $(\varphi \vee \psi) \triangleright T$ and (for reductio) $\neg(\varphi \triangleright T)$ and $\neg(\psi \triangleright T)$. Thus (CV) $(\varphi \vee \psi) \triangleright \neg(\varphi \vee$ $\neg \psi)$, hence $(\varphi \vee \psi) \triangleright(\psi \wedge \neg \varphi)(\mathrm{RCM})$. Similarly deduce $(\varphi \vee \psi) \triangleright(\neg \psi \wedge \varphi)$. This implies $(\varphi \vee \psi) \triangleright \perp(\mathrm{CC}, \mathrm{RCM})$, contradicting (Con).

44 If we replace CMon by CSO, CA2 is redundant (see footnote 36 ).

45 Generally, a neutral conditional is definable from a standard conditional by $\varphi \triangleright \psi \equiv((\varphi>$ $\psi) \wedge \neg(\varphi>\perp)$ ). This is equivalent to Lewis' (1973, p. 438) definition of $\varphi \square \Rightarrow \psi$ which he interprets deontically, temporally, and egocentrically. 


$$
\begin{aligned}
& \neg(\varphi \triangleright \perp) \\
& \varphi \triangleright \top \\
& (\neg(\varphi \triangleright \varphi) \wedge(\varphi \triangleright \psi)) \rightarrow(\perp \triangleright \psi) \\
& ((\varphi \triangleright \chi) \wedge \neg(\psi \triangleright \psi)) \rightarrow(\neg(\perp \triangleright \chi) \rightarrow((\varphi \vee \psi) \triangleright \chi)) \\
& (\perp \vee \psi) \rightarrow(\varphi \triangleright \psi) \\
& ((\varphi \vee \psi) \triangleright(\varphi \vee \psi)) \rightarrow((\varphi \triangleright \varphi) \vee(\psi \triangleright \psi)) \\
& ((\varphi \wedge \psi) \triangleright(\varphi \wedge \psi)) \rightarrow(\varphi \triangleright \varphi) \\
& (\perp \triangleright(\varphi \rightarrow \psi)) \rightarrow((\perp \triangleright \varphi) \rightarrow(\perp \triangleright \psi)) \\
& (\perp \triangleright \varphi) \rightarrow \varphi \\
& \neg(\perp \triangleright \neg \varphi) \rightarrow(\perp \triangleright \neg(\perp \triangleright \neg \varphi))
\end{aligned}
$$

Proof. Theorem 7.18 and Corollary 7.21.

This axiomatisation also drops ID, by imposing Con, but restores $\mathrm{CN}$ and comes with new axioms which handle two implicit modalities-the new outer doxastic possibility $\checkmark \varphi \equiv \varphi \triangleright \varphi^{46}$ and the absolute (or metaphysical) necessity $\boxminus \varphi \equiv \perp \triangleright \varphi .^{47}$ Metaphysical necessity implies doxastic necessity $\square$ (by $\mathrm{C}, \mathrm{CC}, \mathrm{Con}$ ). $\mathrm{E}$ and $\mathrm{F}$ amount to being closed under conjunction and upwards closed (Con $+\mathrm{CN}$ establish that $\square$ has the remaining filter properties). $\mathrm{K}^{*}, \mathrm{~T}^{*}$ and $5^{*}$ are the obvious Kripke axioms for $\boxminus$, imposing that metaphysical necessity is knowledge-like, i.e., closed under modus ponens, factual and negatively introspectible and thus also positively introspectible. A, B and $\mathrm{C}$ are interaction axioms. $\mathrm{C}$ says that conditionals with metaphysically necessary consequents are true. A says that true conditionals have either a doxastically possible antecedent or a metaphysically necessary consequent. B is equivalent (by A, C) to CA2. Thus the antecedent in a conditional can be disjunctively weakened by a doxastically impossible proposition. For regular ranking functions, $\boxminus$ collapses to $\square$ (by which $E$ and F become redundant) and ID holds, except for $\varphi \equiv \perp$.

As opposed to the neutral conditional, the doxastic conditional restores all ignorant conditionals $\varphi>\top(\mathrm{CN})$ and has the means to model a knowledge-like (KT5) necessity $\boxminus$ in addition to the belief-like (KD) outer modality $\square$. Thus the doxastic conditional requires one axiom involving a nested conditional $\left(5^{*}\right) .{ }^{48}$

The above logic is interesting in view of the following result (Unterhuber, 2016, Theorem 11): any normal conditional logic (PT, MoPo, RCEA, RCM, CC, CN) which contains AT $\neg(\varphi>\neg \varphi)$ is either inconsistent or incomplete in Chellas (!) semantics. ${ }^{49}$ However, the above logic is normal, can be shown to contain $\mathrm{AT}^{50}$ and yet it is consistent and complete in the doxastic ranking semantics.

\subsection{Metaphysical ranking semantics.}

THEOREM 5.3. VT5 is sound and complete for the metaphysical ranking semantics.

Proof. Theorem 7.24 and Corollary 7.27.

46 Doxastic possibility again reduces semantically to $\kappa^{w}(\varphi)<\infty$. But now $\varphi>\varphi$ differs from $\varphi \triangleright \mathrm{T}$.

47 Semantically, $\varphi$ is metaphysically necessary in $\mathfrak{R}$ iff $\models_{\mathfrak{R}} \varphi$.

48 Generally, a doxastic conditional is definable from a standard conditional and an 'absolute' necessity $\boxminus$, provided (IA) $\boxminus \varphi \rightarrow(\neg \varphi>\perp)$, by $\varphi \triangleright \psi \equiv((\varphi>\psi) \wedge \neg(\varphi>\perp)) \vee(\boxminus \psi \wedge(\varphi>$ $\perp)$ ).

49 Chellas' models are essentially selection models.

50 Suppose $\varphi \triangleright \neg \varphi$. Either $\varphi \triangleright \varphi$ or $\neg(\varphi \triangleright \varphi)$. In the first case $\varphi \triangleright \perp$ (CC), thus $\perp$ (Con), in the second case $\perp \triangleright \perp$ (CMon) thus $\perp$ ( $\left.\mathrm{T}^{*}\right)$. Hence $\neg(\varphi \triangleright \neg \varphi)$. 
This is not astonishing, since metaphysical conditionalisation preserves all features of standard conditionalisation (i.e., conserving ranking-differences within $A$ ) and acts as if the ranking functions were regular. We therefore have three different ranking semantics for VT5: the reflexive-euclidean ranking semantics, the sub-semantics of regular models and the metaphysical ranking semantics. Hence, on the level of validity, it makes no difference, whether we impose the semantic properties ( $\mathrm{t}$ ) and (v) on rankings, whether we consider them to be regular (i.e., dynamically open-minded) or whether we change abnormal conditionalisation to metaphysical conditionalisation. However, on the level of truth, the outer modality of the metaphysical and the regular ranking semantics represents an absolute necessity $\boxminus$ (with underlying $R=W^{2}$ ), whereas for a (t,v)-ranking semantics it represents just a knowledge-like necessity ( $R$ is an equivalence relation). Contrary to the neutral and the doxastic ranking semantics, the metaphysical ranking semantics rejects contradictory conditionals $\varphi>\perp$, except when the antecedent $\varphi$ is equivalent to a contradiction (in the model). And as for the doxastic ranking semantics, one axiom with a nested conditional is required (5).

5.4. Alternative ranking semantics. ${ }^{51}$ An alternative truth definition was suggested by Huber (2017, footnote 4). The idea is that if $\kappa^{w}(\varphi) \geq \kappa^{w}(w)$, the closest $\varphi$-worlds (according to $\kappa^{w}$ ) should be selected, and the sufficiently close $\varphi$-worlds otherwise, where sufficient closeness means at least as close as $\kappa^{w}(w)$. In short, instead of selecting $\left[\kappa^{w}(\varphi)\right]_{\kappa} \cap$ $[\varphi]$, one selects $\left[\leq \max \left\{\kappa^{w}(w), \kappa^{w}(\varphi)\right\}\right]_{\kappa} \cap[\varphi]$, where $[\leq x]_{\kappa}:=\{w \in W: \kappa(w) \leq x\}$. This yields

$$
\text { (A) } w \vDash_{\mathfrak{R}}^{*} \varphi>\psi \quad \text { iff } \quad\left[\leq \max \left\{\kappa^{w}(w), \kappa^{w}\left([\varphi]^{\mathfrak{R}}\right)\right\}\right]_{\kappa^{w}} \cap[\varphi]^{\mathfrak{R}} \subseteq[\psi]^{\mathfrak{R}} \text {. }
$$

(alternative conditional)

If $\kappa^{w}(w)=\infty$ (or $\left.\kappa^{w}([\varphi])=\infty\right)$, the conditional becomes very demanding. In fact, it transforms into a strict implication, since $\varphi>\psi$ then means that all $\varphi$-worlds are $\psi$-worlds.

THEOREM 5.4. VW5 is sound and complete for the alternative ranking semantics.

Proof. See p. 888.

Since 5 is the only nested axiom, the logic in the non-nested fragment will be indistinguishable from VW for that fragment, as Huber (2017) conjectured.

One may want to combine the above alternative definition with either the standard, the neutral, the doxastic or the metaphysical clause. ${ }^{52}$ As an example, let us indicate how to do this for the standard ranking semantics, where we obtain

(1A) $w \vDash_{\mathfrak{R}}{ }^{*} \varphi>\psi$ iff $\kappa^{w}([\varphi])=\infty$ or $\kappa_{[\varphi]}^{w}([\neg \psi])>\max \left\{0, \kappa^{w}(w)-\kappa^{w}([\varphi])\right\}$.

(alternative standard conditional)

Consider the axiom:

$$
\neg(\varphi>\perp) \rightarrow((\varphi>\psi) \rightarrow(\varphi \rightarrow \psi))
$$

Intuitively this means that if $\varphi$ is possible in $w$, then $\kappa^{w}$ behaves as if it were weakly centered.

51 This subsection was added for reasons of exhaustiveness. I thank an anonymous referee for suggesting to treat this case, and Franz Huber for earlier discussions on this idea.

52 Replacing only the disjunct for $\kappa\left([\varphi]^{\mathfrak{R}}\right)<\infty$ by the above alternative clause, and conserving the other disjunct. 
THEOREM 5.5. VP $+\mathrm{MP}^{\diamond}$ is sound and complete for the alternative standard ranking semantics.

Proof. See p. 888.

By a similar reasoning, the complete and sound logic for the alternative neutral ranking semantics should then be $A X n$, augmented by

$$
(\varphi \triangleright T) \rightarrow((\varphi \triangleright \psi) \rightarrow(\varphi \rightarrow \psi))
$$

The complete and sound logic for the alternative doxastic ranking semantics should be AXd, augmented by

$$
(\varphi \triangleright \varphi) \rightarrow((\varphi \triangleright \psi) \rightarrow(\varphi \rightarrow \psi))
$$

And the complete and sound logic for the alternative metaphysical ranking semantics is VWT5 = VW5, since the alternative metaphysical truth condition is equivalent to the above truth condition (A). ${ }^{53}$

§6. Conclusion. This article has extended the analysis of (standard) ranking semantics (Friedman \& Halpern, 2001) in three directions: First, by allowing ranking functions to not have their minimum at 0 , we obtained that Lewis' $V$ is complete for this generalised ranking semantics. Second, I showed that the Lewisian hierarchy of conditional logicsinvolving axioms such as MP, CS, CEM, or T, 4, 5 for the outer modality, as well as the postulate $\mathrm{U}$ (comparable to Lewis' absoluteness) — can be reproduced in the ranking semantics by imposing corresponding properties on the ranking functions. As a consequence these logics, which are often understood as logics for ontic (or counterfactual) conditionals, can in principle be reinterpreted doxastically. As corollaries, we obtained the completeness of VP for the ranking semantics, of VT5 for Huber's (2014) regular ranking semantics and of VPU for Spohn's (2015) global-carnapian ranking semantics. When restricting to the non-nested conditional language, the latter two semantics are indistinguishable from VT and VP, respectively, correcting the claimed completeness of V in Huber (2014) and Spohn (2015). The third direction investigated modifications of the trivial-truth clause for impossible-antecedent conditionals, as suggested by Huber (2014, 2015, 2016).

A standard conditional is trivially true for an impossible antecedent. But one might also set it to be always false (neutral conditional), to be true or false depending on whether the consequent is absolutely necessary (doxastic conditional), or depending on whether the antecedent implies the consequent (metaphysical conditional).

In addition to the semantic considerations, I also provided a doxastic and an axiomatic argument for these modifications: The doxastic argument was based on interpreting the conditional as a conditional belief induced by ranking conditionalisation. The standard conditional then corresponds to believing everything when abnormally conditionalising on a doxastically impossible proposition, the neutral conditional to believing nothing, the doxastic conditional to believing only what is absolutely necessary, and the metaphysical conditional to believing only what follows from the antecedent. Correspondingly, abnormal conditionalisation (corresponding to the standard conditional) is AGM only over regular

53 Because when $\kappa^{w}(\varphi)=\infty$, the condition $\left[\leq \max \left\{\kappa^{w}(w), \kappa^{w}(\varphi)\right\}\right]_{\kappa} \cap[\varphi] \subseteq[\psi]$ is equivalent to $[\varphi] \subseteq[\psi]$. 
ranking functions and else violates consistency. The metaphysical conditionalisation is fully AGM, the doxastic conditionalisation violates success and the neutral conditionalisation violates success and consistency.

The modifications affect the logic. The metaphysical conditional rejects $\varphi>\perp$ except for contradictory antecedents $\varphi$, has the sound and complete logic VT5 and thus agrees with the regular ranking semantics. The neutral and the doxastic semantics reject all contradictory conditionals $\varphi>\perp$ and thus drop ID. In both semantics the antecedent of a conditional can be disjunctively weakened by an impossible proposition (CA2 or B). The neutral ranking semantics also drops CN, restores ID for the tautology (ID2) and for doxastically possible antecedents (ID1), and requires the dual doxastic necessity to be closed under conjunction (CMon2), consistent and upwards closed. The doxastic ranking semantics additionally models a knowledge-like metaphysical necessity which implies the outer doxastic necessity. It also requires that a conditional with metaphysically necessary consequent is true $(\mathrm{C})$ and that a true conditional has either a doxastically possible antecedent or a metaphysically necessary consequent (A).

Finally, I also analysed an alternative ranking semantics, in which $\varphi>\psi$ in $w$ iff $\psi$ in the sufficiently closest $\varphi$-worlds, where sufficient closeness is maximal closeness if $\varphi$ is more implausible than $w$ from the perspective of $\kappa^{w}$, and else sufficient closeness means to be at most as implausible as $w$. The resulting logic is VW5 $\equiv$ VWT5, which strengthens the logic VT5 by the axiom MP. I then implemented this alternative ranking semantics within the different above variations. For the standard, neutral and doxastic alternative ranking semantics this lead to a weakening of the axiom MP, which needed to be restricted to the cases where the antecedent is already possible.

The ranking-theoretic simulation of the Lewisian hierarchy suggests that a doxastic reinterpretation of so-called ontic conditionals is in principle possible. The philosophical consequences of this could not be analysed here. ${ }^{54}$ It remains for example an open question whether the ranking-theoretic (or more generally, plausibilistic) reinterpretation of the Lewisian similarity can avoid those problems which are due to Lewis' understanding of similarity and his world-internal determinism, such as the future-similarity objection (Fine, 1975). Furthermore, the following questions remain: How do the new conditionals behave when generated from standard conditionals in weaker logics than VP (see footnote 29)? How does the conditional logic change if ranking-theoretic belief is defined as sufficient disbelief in the negation (cf. p. 3)? What is to be gained when ranking functions are properly ordinal-valued (see footnote 30 ), enabling to define realms of doxastic impossibility? And how should the semantic framework be extended in order to explicitly treat in the object language the tenses and moods appearing in conditionals?

\section{§7. Appendix}

7.1. Related work. Friedman \& Halpern (2001) consider ranking functions $\kappa^{w}$ over $W_{w} \subseteq W$ and define ${ }^{55}$

$$
w, \mathfrak{R} \vDash \varphi>\psi \text { iff } \kappa^{w}(\varphi \wedge \psi)<\kappa^{w}(\varphi \wedge \neg \psi) \text { or } \kappa^{w}(\varphi)=\infty .^{56}
$$

\footnotetext{
54 A philosophical analysis of the ranking-theoretic simulation of Lewis' hierarchy and a discussion of the here presented counter-doxastic conditionals in scientific settings is attempted in Raidl \& Skovgaard-Olsen (2017).

55 Cf. Halpern $(2003,311)$.

56 Adapted notation.
} 
Huber (2014) defines

$$
w, \mathfrak{R} \vDash \varphi>\psi \text { iff }[0]_{\kappa_{\varphi}^{w}} \subseteq[\psi] \quad\left[\text { or } \kappa^{w}([\varphi])=\infty\right] .{ }^{57}
$$

A last reformulation yields

(1c) $w, \mathfrak{R} \vDash \varphi>\psi$ iff $\left[\kappa^{w}(\varphi)\right]_{\kappa^{w}} \cap[\varphi] \subseteq[\psi]$ or $\kappa^{w}([\varphi])=\infty$.

Here $[x]_{\kappa}:=\{w \in W: \kappa(w)=x\}$. In a ranked model, all are equivalent to (1) from $\mathrm{p}$. 865, for (1a), provided one extends $\kappa^{w}$ to $W$, setting $\kappa^{w}(v)=\infty$, when $v \in W \backslash W_{w}$.

Define a selection function by $F(w, \varphi):=\left[\kappa^{w}(\varphi)\right]_{\kappa^{w}} \cap[\varphi]$ if $\kappa^{w}(\varphi)<\infty$, and else $F(w, \varphi):=\emptyset$. (1c) is then equivalent to the truth clause for the selection model $\mathfrak{M}=$ $\langle W, F, V\rangle$ :

(1d) $\quad w, \mathfrak{M} \vDash \varphi>\psi$ iff $F(w, \varphi) \subseteq[\psi]$.

Spohn $(2015,10-11)$ considers $W$ to be all truth functions over $\operatorname{Var}(\mathcal{L})$, with $\langle w, \kappa\rangle \vDash p$ iff $w(p)=1$, and defines (for $\varphi, \psi$ conditional-free):

(1e) $\langle w, \kappa\rangle \vDash \varphi>\psi$ iff $\kappa_{[\varphi]}(\overline{[\psi]})>0$.

Semi-epistemic validity is defined as $\models^{s e} \chi$ iff $\langle w, \kappa\rangle \vDash \chi$ for all $w \in W$ and all ranking functions $\kappa$. However, (1e) is incomplete, since it is not specified what happens if $\kappa([\varphi])=$ $\infty$. There are at least two options: (1e.1) use the standard trivial truth condition (adding 'or $\kappa([\varphi])=\infty$ ' on the right side of $(1 \mathrm{e}))$, or (1e.2) if $\kappa_{[\varphi]}$ is undefined assume not $\kappa_{[\varphi]}(\overline{[\psi]})>$ 0 , by which $(1 \mathrm{e})$ reduces to the neutral conditional (i.e., add 'and $\kappa([\varphi])<\infty$ ' on the right side of (1e)).

7.2. Selection models. $\mathfrak{M}=\langle W, F, V\rangle$ is a selection model for $\operatorname{Var}=\operatorname{Var}\left(\mathcal{L}_{>}\right)$ iff (1) $W \neq \varnothing$ is a set of worlds, (2) $V: \operatorname{Var} \longrightarrow \wp(W)$ is a valuation, (3) $F: W \times$ $\wp(W) \longrightarrow \wp(W)$ is an (extensional) selection function, i.e., total over $W \times\left\{[\varphi]^{\mathfrak{M}}: \varphi \in\right.$ $\left.\mathrm{S}\left(\mathcal{L}_{>}\right)\right\}$, where standard connectives are defined as usual and $>$by

- $\quad w, \mathfrak{M} \vDash \varphi>\psi$ iff $F\left(w,[\varphi]^{\mathfrak{M}}\right) \subseteq[\psi]^{\mathfrak{M}}$.

$F$ is a total selection function iff it is total over $\wp(W)$.

A conditional logic $\Sigma$ is normal iff $\Sigma$ contains $\mathrm{CN}$ and is regular, i.e., contains PT, $\mathrm{CC}$ and is closed under MoPo, RCEA and RCM. ${ }^{58}$ Write CK the smallest normal conditional logic and $\mathrm{CKX}_{1} \ldots \mathrm{X}_{n}$ the smallest normal conditional logic containing axioms $\mathrm{X}_{1}, \ldots, \mathrm{X}_{n}$.

THEOREM 7.1. CK is sound and complete for selection models.

Proof. Cf. Chellas (1975). Soundness: PT, CC, CN are valid and MoPo, RCEA, RCM preserve validity. Completeness, by a canonical model construction: Let $\Sigma$ be a normal conditional logic. Its canonical model is $\mathfrak{M}^{\Sigma}=\left\langle W^{\Sigma}, F^{\Sigma}, V^{\Sigma}\right\rangle$, where $W^{\Sigma}$ are the maximal $\Sigma$-consistent theories $V^{\Sigma}(p)=\left\{w \in W^{\Sigma}: p \in w\right\}$ and $v \in F^{\Sigma}(w,[\varphi])$ iff $\left\{\psi \in \mathrm{S}\left(\mathcal{L}_{>}\right): \varphi>\psi \in w\right\} \subseteq v$ (well defined by RCEA). We have: (1) $\mathfrak{M}^{\Sigma}$ is a selection model $(\emptyset$ is $\Sigma$-consistent and thus has a maximal consistent extension by the Lindenbaum Lemma, the rest by definition), (2) $\varphi>\psi \in w$ iff for every $v$, if $\{\chi: \varphi>\chi \in w\} \subseteq v$ then $\psi \in v$ (normality of $\Sigma$ is needed here), (3) the truth Lemma holds: $w, \mathfrak{M}^{\Sigma} \vDash \varphi$ iff

57 Brackets are added since Huber considers regular ranking functions.

58 For an equivalent definition, see Chellas (1975). 
$\varphi \in w$ (induction, using (2)), (4) $\mathfrak{M}^{\Sigma}$ determines $\Sigma$, i.e., $\mathfrak{M}^{\Sigma} \vDash \varphi$ iff $\vdash_{\Sigma} \varphi\left(\mathfrak{M}^{\Sigma} \vDash \varphi\right.$, thus $w, \mathfrak{M}^{\Sigma} \vDash \varphi$ for all $w$, hence $\varphi \in w$ for all $w(3)$, therefore $\vdash_{\Sigma} \varphi$, since in general: $\Gamma \vdash_{\Sigma} \varphi$ iff $\varphi \in \Delta$ for each maximal $\Sigma$-consistent $\Delta \supseteq \Gamma$ ). Suppose $\nvdash_{C K} \varphi$, then $\mathfrak{M}^{\mathrm{CK}} \not \models \varphi$ (determination), hence $\varphi$ is not valid.

LEMMA 7.2. The canonical model of $\mathrm{CK}_{1} \ldots \mathrm{X}_{n}$ satisfies the corresponding properties $\left(x_{i}\right)$ over sentence classes $^{59}$ :

(1) $F(w, A) \subseteq A$,

(2) $F(w, W) \neq \emptyset$,

(3) if $F(w, A) \subseteq C$ and $F(w, B) \subseteq C$, then $F(w, A \cup B) \subseteq C$,

(4) if $F(w, A) \subseteq C$ and $F(w, A) \subseteq B$, then $F(w, A \cap B) \subseteq C$,

(5) if $F(w, A) \subseteq C$ and $F(w, A) \nsubseteq \bar{B}$, then $F(w, A \cap B) \subseteq C$,

(6) if $F(w, A) \subseteq B$ and $F(w, B) \subseteq A$, then $F(w, A)=F(w, B)$,

(7) if $F(w, A)=\emptyset$, then $w \in \bar{A}$,

(8) if $F(w, A) \neq \emptyset$, then $F(w,\{v \in W: F(v, A)=\emptyset\})=\emptyset$,

(9) if $F(w, A)=\emptyset$, then $F(w,\{v \in W: F(v, A) \neq \emptyset\})=\emptyset$,

(10) if $F(w, A) \subseteq B$, then $w \in \bar{A} \cup B$,

(11) $F(w, A) \subseteq B$ or $F(w, A) \subseteq \bar{B}$,

(12) if $F(w, A) \subseteq B$, then $F(w,\{v \in W: F(v, A) \nsubseteq B\})=\emptyset$,

(13) if $F(w, \bar{A})=\emptyset$ then $F(w, B) \subseteq A$,

(14) if $w \in A$, then $w \in F(w, A)$,

(15) if $w \in A$, then $F(w, A)=\{w\}$.

Additionally $[\mathrm{cw}]$ follows from $(M P)$ and $[\mathrm{cs}]$ from $(M P, C S)$.

Proof. Using (2) and the truth Lemma (3) from Theorem 7.1. E.g:

(p) $\neg(\top>\perp) \in w(\mathrm{P})$, i.e., $(\top>\perp) \notin w$ (consistency). Thus not all $v$ extending $\{\varphi: \top>\varphi \in w\}$ have $\perp \in v$. Hence there is a consistent extension, i.e., $F(w,[\top]) \neq \emptyset$.

(u) Suppose $F(w,[\varphi]) \subseteq[\psi]$. Then $\varphi>\psi \in w$. Thus $\neg(\varphi>\psi)>\perp \in w$ (U). Therefore $F(w,\{v: F(v,[\varphi]) \nsubseteq[\psi]\})=\emptyset$.

(cw) from MP: Suppose $\varphi \in w . \varphi>\psi \in w$ implies $\varphi \rightarrow \psi \in w$ (MP). Thus, if $\varphi>\psi \in w$, then $\psi \in w$ (since $\varphi \in w$ ). Hence $w$ extends $\{\psi: \varphi>\psi \in w\}$, i.e., $w \in F(w,[\varphi])$.

(cs) from MP and CS: Suppose $\varphi \in w$, i.e., $w \in F(w,[\varphi])$ (cf., cw). Assume (for reductio) that there is $v \in F(w,[\varphi])$, such that $v \neq w$. Then there is $\psi$, such that $\psi \in v$ but $\psi \notin w$, i.e., $\neg \psi \in w$, hence $(\varphi \wedge \neg \psi) \in w$. Thus $\varphi>\neg \psi \in w$ (CS). But then $\neg \psi \in v$, contradicting $\mathrm{CK}+\mathrm{MP}+\mathrm{CS}$-consistency.

If $\mathfrak{M}$ is an (id, ca, cmon) selection model then

59 Properties are quantified over $w \in W$ and classes $A=[\varphi], B=[\psi], C=[\chi]$, where one verifies that $W=[\top], \varnothing=[\perp], \bar{B}=[\neg \psi], A \cup B=[\varphi \vee \psi], A \cap B=[\varphi \wedge \psi],\{v \in W: F(v, A)=$ $\emptyset\}=[\varphi>\perp]$.

60 Properties with no corresponding direct axiomatisation are written $[\mathrm{x}]$ instead of $(\mathrm{x})$. 
- $F(w,[\varphi])=\emptyset$ iff $R(w) \cap[\varphi]=\emptyset$.

By RCEA, we may write $F(w, \varphi)=F(w,[\varphi])$.

COROLLARY 7.3. The canonical model of $\mathrm{CK}+\mathrm{ID}+\mathrm{CA}+\mathrm{CMon}+\mathrm{U}$ satisfies

- if $v \in R(w)$, then $F(w, A)=F(v, A)$.

Proof. Suppose $v \in R(w)$ and $\varphi>\psi \in w$. Thus $F(w, \varphi) \subseteq[\psi]$. Therefore $F(w, \neg(\varphi>$ $\psi))=\emptyset(\mathrm{u})$. Hence, $\varphi>\psi \in v[\mathrm{a}]$. Assume now that $u \in F(v, \varphi)$. Then $\{\psi: \varphi>\psi \in$ $v\} \subseteq u$, hence $\{\psi: \varphi>\psi \in w\} \subseteq u$, i.e., $u \in F(w, \varphi)$. Thereby $F(v, \varphi) \subseteq F(w, \varphi)$. (U) implies (u), which implies (iv). Thus, since $v \in R(w)$, we have $w \in R(v)$, leading to $F(w, \varphi) \subseteq F(v, \varphi)$.

A selection model is Lewisian iff it satisfies (id, ca, cv, cmon) $)^{61}$ and ranky iff it satisfies additionally (p).

COROLLARY 7.4. CK $\mathrm{X}_{1} \ldots \mathrm{X}_{n}$ is sound and complete for the $x_{1}, \ldots x_{n}$-selection models. In particular, in the following table, the logic on the left is sound and complete for the class of selection models on the right:

\begin{tabular}{l|lll} 
Logic & Frame Property & Name & Equivalent \\
\hline V & id, ca, cmon, cv & Lewisian & \\
VP & id, ca, cmon, cv, p & ranky & (p)-Lewisian \\
VT & id, ca, cmon, cv, t & (t)-ranky & (t)-Lewisian \\
VW & id, ca, cmon, cv, cw & (cw)-ranky & (cw)-Lewisian \\
VC & id, ca, cmon, cv, cw, cs & (cw, cs)-ranky & (cw, cs)-Lewisian \\
C2 & id, ca, cmon, cv, t, cem & (t,cem)-ranky & (t, cem)-Lewisian
\end{tabular}

Proof. Soundness is clear (note cw implies mp, which implies t which implies p). Completeness: by Lemma 7.2 with the argument in Theorem 7.1.

LEMMA 7.5. MOD $(\neg \varphi>\varphi) \rightarrow(\psi>\varphi)$ follows from $\mathrm{CK}+\mathrm{ID}+\mathrm{CA}+\mathrm{CMon}$.

Proof. Assume $\neg \varphi>\varphi$. But $\neg \varphi>\neg \varphi$ (ID), thus $\neg \varphi>\perp$ (CC), i.e., $\neg \varphi>\psi$ (RCM). Therefore $(\neg \varphi \wedge \psi)>\perp$ (CMon), i.e., $(\neg \varphi \wedge \psi)>\varphi ;(\varphi \wedge \psi)>\varphi$ (ID, RCM) and thus $\psi>\varphi$ (RCEA, CA).

LEMMA 7.6. CMon and $\mathrm{CSO}$ are equivalent, given $\mathrm{CK}+\mathrm{ID}+\mathrm{CA}$.

Proof. $(\Leftarrow)$ Assume $\varphi>\chi, \varphi>\psi$. Thus $\varphi>(\varphi \wedge \psi)($ ID, CC). But $(\varphi \wedge \psi)>\varphi$ (ID, RCM). Thus $(\varphi \wedge \psi)>\chi(\mathrm{CSO})$. $(\Rightarrow)$ Suppose $\varphi>\psi, \psi>\varphi$ and $\varphi>\chi$. Thus $(\varphi \wedge \psi)>\chi(\mathrm{CMon})$ and $(\varphi \wedge \psi)>\psi(\mathrm{ID}, \mathrm{RCM})$. Hence $(\varphi \wedge \psi)>(\chi \wedge \psi)(\mathrm{CC})$. But $(\neg \varphi \wedge \psi)>(\neg \varphi \wedge \psi)$ (ID). Hence $\psi>((\chi \wedge \psi) \vee(\neg \varphi \wedge \psi))$ (RCM, RCEA, CA). Yet $\psi>\varphi$, thus $\psi>((\chi \wedge \psi) \wedge \varphi)(\mathrm{CC}, \mathrm{RCM})$. Therefore $\psi>\chi(\mathrm{RCM})$.

7.3. Generalised and standard ranking semantics. Let $\mathfrak{M}=\langle W, \ldots, V\rangle, \mathfrak{M}^{\prime}=$ $\left\langle W^{\prime}, \cdots, V\right\rangle$ be two models for $\mathrm{S}\left(\mathcal{L}_{>}\right)$, possibly with different interpretations for $>$, and $W^{\prime} \subseteq W . \mathfrak{M}^{\prime}$ is faithful to $\mathfrak{M}$ iff for all $\varphi$ and all $w \in W^{\prime}, w, \mathfrak{M}^{\prime} \vDash \varphi$ iff $w, \mathfrak{M} \vDash \varphi$.

61 Equivalent to Lewis' (1971) $\alpha$-models. (id) is Lewis' ( $\alpha .0 .6)$, (cmon) is equivalent to $(\mathrm{cso})=(\alpha .0 .7)$, given $(\mathrm{id}, \mathrm{ca})$, and $(\mathrm{ca}, \mathrm{cv})$ is equivalent to $(\alpha .0 .8)$. 
Proof of Theorem 3.6. For the terminology on selection models, see $\$ 7.2{ }^{62}$

Soundness: Let $\mathfrak{R}=\left\langle W,\left(\kappa^{w}\right)_{w \in W}, V\right\rangle$ be a generalised ranked model. Define $F(w, A)=\left[\kappa^{w}(A)\right]_{\kappa^{w}} \cap A$ if $\kappa^{w}(A)<\infty$ and $=\emptyset$ otherwise. Then $\mathfrak{M}(\mathfrak{R}):=\langle W, F, V\rangle$ is a Lewisian (id, ca, cv, cmon) selection model and faithful to $\mathfrak{R}$. Since $\mathfrak{M}(\mathfrak{R})$ validates $\mathrm{V}$ (Corollary 7.4), $\mathfrak{R}$ does as well (faithfulness). If $\mathfrak{R}$ is a standard ranked model, then $\mathfrak{M}(\mathfrak{R})$ is ranky (id, ca, cv, cmon, p). Then $\mathfrak{M}(\mathfrak{R})$ validates VP (Corollary 7.4) and thus $\mathfrak{R}$ does as well (faithfulness).

Completeness: Suppose $\nvdash_{\Sigma} \varphi$ (where $\Sigma=\mathrm{V}$ ). Then there is a (canonical) Lewisian selection model $\mathfrak{M}=\mathfrak{M}^{\Sigma}$ such that $\mathfrak{M} \not \neq \varphi$ (Theorem 7.1, Lemma 7.2). Thus there is a $\varphi$-filtration $\mathfrak{M}^{*}$ of $\mathfrak{M}$ such that $\mathfrak{M}^{*} \not \models \varphi$ (cf. Lewis (1971)). $\varphi$-filtrations preserve truth (in the reduced language) and properties, i.e., $\mathfrak{M}^{*}$ remains Lewisian. Additionally, it is finite, nonredundant (two worlds satisfying the same propositional variables are the same world) and the selection function is total. From $\mathfrak{M}^{*}=\langle W, F, V\rangle$ one constructs $\mathfrak{R}\left(\mathfrak{M}^{*}\right):=$ $\left\langle W,\left(\kappa^{w}\right)_{w \in W}, V\right\rangle$, by defining $R(w):=\bigcup_{A \in \wp(W)} F(w, A),[\infty]:=W \backslash R(w)$; and if $R(w) \neq \emptyset$ then recursively:

- $W_{0}:=R(w),[0]:=F\left(w, W_{0}\right)$,

- $W_{n+1}:=W_{n} \backslash[n],[n+1]:=F\left(w, W_{n+1}\right)$ if $[n] \neq W_{n}$. If $[n]=W_{n}$ stop,

- $\kappa^{w}(\{v\})=x$ if $v \in[x]$, and $\kappa^{w}(A)=\min _{v \in A} \kappa^{w}(\{v\})$ and $\kappa^{w}(\emptyset)=\infty$.

Note: $F(w,[\infty])=\emptyset$ (otherwise $R(w) \cap[\infty] \neq \emptyset$, contradicting $[\infty]=W \backslash R(w)$ ). One shows that (1) $\mathfrak{R}\left(\mathfrak{M}^{*}\right)$ is a generalised ranked model, where $\kappa^{w}$ is a standard ranking function if $R(w) \neq \emptyset$ and else $\kappa^{w}(A)=\infty$ for all $A$. Additionally, (2) for all $w \in W$ and all $A \in \wp(W)$ we have $F(w, A)=\left[\kappa^{w}(A)\right]_{\kappa^{w}} \cap A$ if $F(w, A) \neq \emptyset$ (and then $\left.\kappa^{w}(A)<\infty\right)$, and else $\kappa^{w}(A)=\infty$. (3) $\mathfrak{R}\left(\mathfrak{M}^{*}\right)$ is faithful to $\mathfrak{M}^{*}$. Thus $\mathfrak{R}\left(\mathfrak{M}^{*}\right) \not \models \varphi$ (3). Therefore $\not \models \varphi$ in the generalised ranking semantics (1).

Let us show (2): if $F(w, A)=\emptyset$, then $R(w) \cap A=\emptyset$ (a). Therefore $\kappa^{w}(A)=$ $\infty$. Conversely, if $\kappa^{w}(A)=\infty$ then $A \subseteq[\infty]=W \backslash R(w)$ (definition), hence $A \cap$ $R(w)=\emptyset$, i.e., $F(w, A)=\emptyset($ a). Suppose $F(w, A) \neq \emptyset$. Since $F(w, A) \subseteq R(w)$, there is a first $n$, such that $[n] \cap A \neq \emptyset$. Since $[n]=\left[\kappa^{w}(A)\right]_{\kappa^{w}}$ it suffices to prove $F(w, A)=[n] \cap A$.

(ᄃ) Split $A^{\prime}=A \cap R(w)$ and $A^{\prime \prime}=A \cap[\infty]$. Since $[n]=F\left(w, W_{n}\right)$ and $[n] \cap A^{\prime}=$

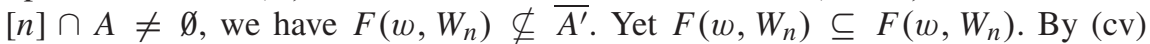
$F\left(w, W_{n} \cap A^{\prime}\right) \subseteq F\left(w, W_{n}\right)$. But $A^{\prime} \subseteq W_{n}$ and thus $F\left(w, A^{\prime}\right) \subseteq F\left(w, W_{n}\right)$. By $F\left(w, A^{\prime}\right) \subseteq A^{\prime}(\mathrm{id})$, we have $F\left(w, A^{\prime}\right) \subseteq[n] \cap A^{\prime}=[n] \cap A$. But $F\left(w, A^{\prime \prime}\right)=$ $\emptyset \subseteq[n] \cap A$ and thus $F(w, A) \subseteq[n] \cap A$ (ca).

(つ) Every $F(w, B)$ is a fixed point (for $B \subseteq R(w)): F(w, F(w, B)) \subseteq F(w, B) \subseteq B$ (id) and $F(w, B) \subseteq F(w, B)$. Thus $F(w, B)=F(w, F(w, B))$ (cso; by cmon, id, ca). $[n] \cap A$ is also a fixed point, $F(w,[n] \cap A)=[n] \cap A$ : Suppose not (for reductio). Then $F(w,[n] \cap A) \subsetneq[n] \cap A$ (id). Therefore there is $v \in[n] \cap A$ such that $F(w,[n] \cap A) \subseteq[n] \backslash\{v\}$. But $F(w,[n] \cap \bar{A}) \subseteq[n] \cap \bar{A}$ (id) and since $v \notin[n] \cap \bar{A}$, we have $F(w,[n] \cap \bar{A}) \subseteq[n] \backslash\{v\}$. Thus $F(w,[n]) \subseteq[n] \backslash\{v\}$ (ca), i.e., $F(w,[n]) \subsetneq[n]$, contradicting that $[n]=F\left(w, W_{n}\right)$ is a fixed point. Thus $F(w,[n] \cap A)=[n] \cap A$. But $F(w,[n] \cap A) \subseteq A$ and $F(w, A) \subseteq[n] \cap A$ (cf. above). Thus $F(w, A)=F(w,[n] \cap A)($ cso). Therefore $F(w, A)=[n] \cap A$.

62 We cannot use Friedman-Halpern's (2001) result for V, since all their models validate P! 
If $\Sigma=$ VP everything is the same, except that now $\mathfrak{M}$ is a ranky selection model and thus all $\kappa^{w}$ of $\mathfrak{R}\left(\mathfrak{M}^{*}\right)$ are standard $(F(w,[\top]) \neq \emptyset(\mathrm{p})$, thus $R(w) \neq \emptyset(\mathrm{a})$, hence [0] $=$ $F(w, R(w)) \neq \emptyset$, by (ca), because $F(w,[\infty])=\emptyset)$.

Proof of Theorem 3.7. Adapt the above proof to the extended properties. Soundness: show that property $(\kappa x)$ of $\kappa^{w}$ implies property $(x)$ of $F(w,$.$) induced by \kappa^{w}$. Completeness: show that property $(x)$ of $F(w,$.$) which in the canonical model follows from axiom$ $(X)$ (Lemma 7.2) implies property $(\kappa x)$ for $\kappa^{w}$ constructed as above. ${ }^{63}$

Proof of Corollary 3.8. There is a twist for completeness.

Regular: Suppose $\nvdash_{\Sigma} \varphi$, where $\Sigma=$ VT5. For $\mathfrak{M}^{*}=\langle W, F, V\rangle$ a ranky, (t, v)selection model (obtained as $\varphi$-filtration from the canonical model), the relation $R$ induced from $F$ (cf. above) is an equivalence relation on $W$. For $C$ an equivalence cell, define the restriction of $\mathfrak{M}$ to $C, \mathfrak{M}_{C}:=\left\langle C, F_{C}, V_{C}\right\rangle$, in the obvious manner. Then (1) $\mathfrak{M}_{C}$ is a selection model, $F_{C}(w, A)=F(w, A)$ for $w \in C, A \subseteq C$, and $R^{\mathfrak{M}_{C}}(v)=R(v)$ for $v \in C$. (2) $\mathfrak{M}_{C}$ preserves properties of $\mathfrak{M}$ and (3) is faithful to $\mathfrak{M}$. Now reproduce the completeness proof: Since $\mathfrak{M}^{*} \not \models \varphi$, there is $w \in W\left(\mathfrak{M}^{*}\right)$ such that $w, \mathfrak{M}^{*} \not \models \varphi$. Let $C$ be the class of $w$. Thus $\mathfrak{M}_{C}^{*} \not \models \varphi$ and therefore $\mathfrak{R}\left(\mathfrak{M}_{C}^{*}\right) \not \models \varphi$. Since $R^{\mathfrak{M}_{C}^{*}}(w)=R(w)=C=$ $W\left(\mathfrak{M}_{C}^{*}\right)$, all ranking functions are regular (i.e., $[\infty]=\emptyset$ ). Therefore $\not \models \varphi$ in the regular ranking semantics.

Global: Suppose $\nvdash_{\Sigma} \varphi$, for $\Sigma=$ VPU. For $\mathfrak{M}^{*}=\langle W, F, V\rangle$ a ranky (u)-selection model (obtained as a $\varphi$-filtration of the canonical model), the relation $R$ is a serial shiftequivalence relation on $W$ and: if $v \in R(w)$ then $F(w, \varphi)=F(v, \varphi)$ (Corollary 7.3). Consider $w \in W$ and $C=R(w) \cup\{w\}$. Then (1)-(3), cf. above, still hold, and (4) $F^{\mathfrak{M}_{C}^{*}}(w, \varphi)=F^{\mathfrak{M}_{C}^{*}}(v, \varphi)$ for all $w, v \in W\left(\mathfrak{M}_{C}^{*}\right)$ (trivially for $v=w$ and because $v \neq w$ implies $v \in R(w))$. Since $\mathfrak{M}^{*} \not \models \varphi$, there is $w \in W\left(\mathfrak{M}^{*}\right)$ such that $w, \mathfrak{M}^{*} \not \models \varphi$. Set $C=$ $R(w) \cup\{w\}$. Thus $\mathfrak{M}_{C}^{*} \not \models \varphi$ and therefore $\mathfrak{R}\left(\mathfrak{M}_{C}^{*}\right) \not \models \varphi$. Since $F^{\mathfrak{M}_{C}^{*}}(w, \varphi)=F^{\mathfrak{M}_{C}^{*}}(v, \varphi)$, all ranking functions agree, i.e., $\mathfrak{R}\left(\mathfrak{M}_{C}^{*}\right)$ is global. Therefore $\not \models \varphi$ in the global ranking semantics.

Global-carnapian: Enrich the global ranked model $\mathfrak{R}=\mathfrak{R}\left(\mathfrak{M}_{C}^{*}\right)$ from above to obtain a global-carnapian ranked model $\mathfrak{R}^{*}$, where $w, \mathfrak{R}^{*} \not \models \varphi$ still holds.

\subsection{Proofs for impossible-antecedent conditionals.}

DEFINITION 7.7. Let $\mathcal{L}_{\gg}, \mathcal{L}_{>}$be two conditional languages.

(1) A map of $>$ to $>$ is a total function $*: S\left(\mathcal{L}_{>}\right) \longrightarrow S\left(\mathcal{L}_{>}\right)$.

(2) A map is a translation, if $p^{*}=p,(\neg \varphi)^{*}=\neg \varphi^{*},(\varphi \circ \psi)^{*}=\left(\varphi^{*} \circ \psi^{*}\right)$ for $\circ \in\{\wedge, \vee, \rightarrow\}$ and there is $A[p, q] \in S\left(\mathcal{L}_{>}\right)$such that $(\varphi>\psi)^{*}=\left(\varphi^{*}>\psi^{*}\right) \circ$ $A\left[\varphi^{*}, \psi^{*}\right]$.

(3) $>$ is covariantly (and, resp. or) definable in $\mathcal{L}_{>}$iff there is a translation $*$, such that $(\circ=\wedge$, resp. $\circ=\vee$, and $)$ for all models $\mathfrak{M}=\langle W, \ldots, V\rangle$ for $\vDash^{>}$, there is a model $\mathfrak{M}^{*}=\langle W, \cdots, V\rangle$ for $\models^{>}$, such that $w, \mathfrak{M} \vDash^{>} \varphi$ iff $w, \mathfrak{M}^{*} \vDash^{>} \varphi^{*}$.

\subsubsection{Neutral Conditional.}

LEMMA 7.8. The neutral conditional - is covariantly and-definable in the ranking semantics for $\mathcal{L}_{>}$by $(\varphi>\psi)^{*}=\left(\left(\varphi^{*}>\psi^{*}\right) \wedge \neg\left(\varphi^{*}>\perp\right)\right)$.

63 Notation, see Definition 3.5. 
Proof. $\mathfrak{R}^{*}=\mathfrak{R}$. Covariance by induction, e.g:

$$
\begin{array}{rllr}
w, \mathfrak{R} \vDash \varphi \triangleright \psi & \text { iff } & \kappa_{\varphi}^{w}(\neg \psi)>0 \& \kappa^{w}(\varphi)<\infty & \text { (def. }) \\
& \text { iff } & \kappa_{\varphi^{*}}^{w}\left(\neg \psi^{*}\right)>0 \& \kappa^{w}\left(\varphi^{*}\right)<\infty & \text { (IH, covariance) } \\
\text { iff } & w, \mathfrak{R} \models^{>}\left(\varphi^{*}>\psi^{*}\right) \wedge \neg\left(\varphi^{*}>\perp\right) & \text { (def. >) }
\end{array}
$$

LEMMA 7.9. Given $\mathrm{V}-\mathrm{CV}, A[\varphi]=\neg(\varphi>\perp)$ together with $>$ validate:

(1) From $\varphi \leftrightarrow \psi$ infer $A[\varphi] \rightarrow A[\psi]$,

(2) $A[\varphi] \rightarrow A[\varphi \vee \psi]$,

(3) $(\neg(\varphi>\neg \psi) \wedge A[\varphi]) \rightarrow A[\varphi \wedge \psi]$.

Proof. SLE by RCEA for $>$. The contraposition of ACA by (RCM, CMon, RCEA). ACV: Assume $\neg(\varphi>\neg \psi), \neg(\varphi>\perp)$ and suppose (for reductio) $(\varphi \wedge \psi)>\perp$. Then $(\varphi \wedge \psi)>\neg \psi(\mathrm{RCM})$. But $(\varphi \wedge \neg \psi)>\neg \psi$ (ID, RCM). Thus $\varphi>\neg \psi$ (CA, RCEA). Contradiction.

THEOREM 7.10. AXn (see p. 871) is sound for the neutral ranking semantics.

Proof. One shows that the translated axioms are true in the standard $\vDash$ and the translated inference rules preserve truth in $\vDash$, using covariance (Lemma 7.8 and 7.9). E.g:

RCEA Suppose $\mathfrak{R} \vDash \varphi \leftrightarrow \psi, \varphi \backslash \chi$. Then $\mathfrak{R} \vDash \varphi^{*} \leftrightarrow \psi^{*}, \varphi^{*}>\chi^{*} \wedge A\left[\varphi^{*}\right]$. Therefore $\mathfrak{R} \vDash \psi^{*}>\chi^{*}$ (RCEA), $A\left[\psi^{*}\right]$ (SLE). Hence $\mathfrak{R} \vDash(\psi>\chi)^{*}$ and thus $\mathfrak{R} \vDash \psi>\chi$ (covariance). (RCM similarly.)

CC Suppose $w, \mathfrak{R} \vDash \varphi>\psi, \varphi>\chi$. Then $w, \mathfrak{R} \vDash\left(\varphi^{*}>\psi^{*}\right) \wedge A\left[\varphi^{*}\right],\left(\varphi^{*}>\chi^{*}\right) \wedge A\left[\varphi^{*}\right]$. Thus $w, \mathfrak{R} \vDash \varphi^{*}>\left(\psi^{*} \wedge \chi^{*}\right)(\mathrm{CC},>)$. (for CA, CV use ACA, ACV, for CMon use CV, CC.)

$\neg(\mathrm{Con})^{*}$ is a contradiction, (ID1)* is a tautology (by ID), similarly (ID2)* (by ID, P), (CMon2)* (by CMon, RCM) and (CA2)* (by CN, CA).

Conversely: $>$ is covariantly or-definable by $>$ as $(\varphi>\psi)^{\circ} \equiv \neg\left(\varphi^{\circ}>\top\right) \vee\left(\varphi^{\circ} \triangleright \psi^{\circ}\right)$, and the translations are inverses of each other, i.e., $\left(\chi^{*}\right)^{\circ}=\chi$ and $\left(\chi^{\circ}\right)^{*}=\chi$ (proof omitted $^{65}$ ).

THEOREM 7.11. If $\vdash^{>} \varphi$ then $\Vdash \varphi^{\circ}$.

Proof. We show, by induction on the length of the proof, that if there is a proof of $\theta$ in $\mathrm{AX}=\mathrm{VP}(>)$, then there is a proof of $\theta^{\circ}$ in $\mathrm{AX}^{\circ}=\mathrm{AXn}(>)$.

$n=1$ : Thus $\theta$ is PT, ID, CC, CA, CMon, CV or P. We prove that the corresponding $\theta^{\circ}$ can be deduced from $A X^{\circ}$. We assume classical laws of reasoning and we make free use of the deduction Lemma, all of which can be obtained by $\operatorname{MoPo}(\bullet)$ and $\mathrm{PT}(\bullet)$. X (left) refer to axioms in $\mathrm{AX}$, those in the proof $(\mathrm{X})$ to $\mathrm{AX}$ ! E.g:

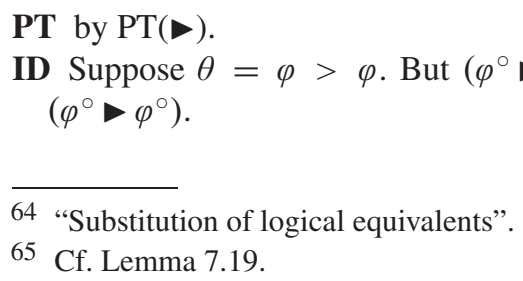


P Suppose $\theta=\neg(\top>\perp)$. But $\neg(\top \triangleright \perp)$ (Con) and $\top \triangleright \top$ (ID2), thus $\neg(\top \triangleright \perp) \wedge$ $(\top \triangleright T)$, i.e., $\neg((\top \triangleright \perp) \vee \neg(\top \triangleright T))=\theta^{\circ}$.

CC Suppose $\theta=((\varphi>\psi) \wedge(\varphi>\chi)) \rightarrow(\varphi>(\psi \wedge \chi))$. Set $A=\varphi^{\circ}>\psi^{\circ}, B=$ $\varphi^{\circ} \triangleright \chi^{\circ}, C=\varphi^{\circ} \vee\left(\psi^{\circ} \wedge \chi^{\circ}\right), D=\neg\left(\varphi^{\circ} \vee \top\right)$. Assume $(A \vee D) \wedge(B \vee D)$, i.e., $(A \wedge B) \vee D$. From $(A \wedge B)$ deduce $C$ (CC) thus $C \vee D$; from $D$ deduce $C \vee D$. Hence, $((A \vee D) \wedge(B \vee D)) \rightarrow(C \vee D)=\theta^{\circ}$

CV, CMon similarly, using (CV, CMon).

CA Suppose $\theta=((\varphi>\chi) \wedge(\psi>\chi)) \rightarrow((\varphi \vee \psi)>\chi)$. Set $A=\varphi^{\circ}>\chi^{\circ}, B=$ $\psi^{\circ} \triangleright \chi^{\circ}, C=\left(\varphi^{\circ} \vee \psi^{\circ}\right) \triangleright \chi^{\circ}, D=\neg\left(\varphi^{\circ} \triangleright \top\right), E=\neg\left(\psi^{\circ} \triangleright \top\right), F=\neg\left(\left(\varphi^{\circ} \vee\right.\right.$ $\left.\left.\psi^{\circ}\right) \triangleright \top\right)$. Suppose $(A \vee D) \wedge(B \vee E)$, yielding four cases: ${ }^{66}$ (11) $A \wedge B$ implies $C$ (CA), thus $C \vee F$. (12) $A \wedge E$ implies $C$ (CA2), (21) $D \wedge B$ similarly. (22) $D \wedge E$ implies $F$, by closure under conjunction of $\mathbf{\square}$, see footnote 43 .

$n+1$. Suppose $\theta_{1}, \ldots, \theta_{n}, \theta_{n+1}$ is a proof of $\theta_{n+1}=\theta$ in AX. We show that there is a proof of $\theta_{n+1}^{\circ}=\theta^{\circ}$ in $\mathrm{AX}^{\circ}$. Our induction hypothesis is that there is a proof of $\theta_{i}^{\circ}$ in $\mathrm{AX}^{\circ}$ for all $i \leq n$.

MoPo clear.

RCEA Suppose $\theta_{n+1}=\psi>\chi$ and there are $\theta_{i}=\varphi \leftrightarrow \psi$ and $\theta_{j}=\varphi>\chi(i, j \leq n)$ and the property holds for $i, j$. Thus (IH) there is a proof of $\theta_{i}^{\circ}=\varphi^{\circ} \leftrightarrow \psi^{\circ}$ and of $\theta_{j}^{\circ}=(\varphi>\chi)^{\circ}=\left(\varphi^{\circ}>\chi^{\circ}\right) \vee \neg\left(\varphi^{\circ}>\top\right)$ in AX. Assume $\varphi^{\circ}>\chi^{\circ}$. Thus $\psi^{\circ}>\chi^{\circ}$ $\left(\right.$ RCEA). Assume $\neg\left(\varphi^{\circ}>\mathrm{T}\right)$. Thus $\neg\left(\psi^{\circ}>\mathrm{T}\right)$ (RCEA). Hence $\theta_{n+1}^{\circ}=(\psi>\chi)^{\circ}$ in both cases.

RCM similarly (using RCM).

THEOREM 7.12. AXn is complete for the neutral ranking semantics.

Proof. Assume $\nvdash_{\mathrm{A} \mathrm{Xn}_{\mathrm{n}}} \theta$, thus $\nvdash_{\mathrm{A} X_{\mathrm{n}}}\left(\theta^{*}\right)^{\circ}\left(*, \circ\right.$ are inverses), hence $\nvdash_{\mathrm{VP}}^{>} \theta^{*}$ (Theorem 7.11). Thus there is a ranked model s.t. $\mathfrak{M} \not \models \theta^{*}$. Hence $\mathfrak{M} \not \nvdash \theta$ (covariance for $\circ$ ).

7.4.2. Doxastic Conditional. Consider a ranked model $\mathfrak{R}$ with doxastic truth relation $\vDash$ and standard truth relation $\vDash$. The absolute ranked model is $\mathfrak{R}^{*}=\langle\mathfrak{R}, R\rangle$, where $R=W^{2}$ defines $\boxminus$ and $\vDash^{*}=\vDash^{\boxminus,>}$ its truth relation.

THEOREM 7.13. The sound and complete axiomatics for absolute ranked models is VP for $>$ and KT5 for $\boxminus$ together with:

- $\boxminus \varphi \rightarrow \square \varphi$

where $\square \varphi=\neg \varphi>\perp$.

Proof. Soundness: clear. Completeness: modify the proof leading to completeness of VP (Theorem 7.1, Corollary 7.4), assuming that $\Sigma$ also contains axiom $\mathrm{K}$ and $\mathrm{N}$ ( $\boxminus \top$ ) for $\boxminus$. Show standard facts such as: for $\Gamma$ maximally $\Sigma$-consistent, with $\Sigma$ containing $\mathrm{K}$ and $\mathrm{N}: \boxminus \varphi \in \Gamma$ iff for every maximally $\Sigma$-consistent $\Delta,\{\psi: \boxminus \psi \in \Gamma\} \subseteq \Delta$ implies $\varphi \in \Delta$.

The canonical model (cf. Theorem 7.1) is enriched by $\langle w, v\rangle \in R^{\Sigma}$ iff $\{\varphi: \boxminus \varphi \in w\} \subseteq$ $v$. Then $\mathfrak{M}^{\Sigma}=\left\langle W^{\Sigma}, F^{\Sigma}, R^{\Sigma}, V^{\Sigma}\right\rangle$ is a ranky selection model, augmented by a Kripke

66 For a conjunction of two disjunctions we denote $i j$ the conjunction of the $i$-th disjunct in the first conjunct and the $j$-th disjunct in the second conjunct. Similarly for longer conjunctions of disjunctions. 
relation. One shows that if $\Sigma$ contains the axioms T, 5 for $\boxminus$, then $R^{\Sigma}$ is reflexive and euclidean and if $\Sigma$ contains IA, then $R_{F}(w) \subseteq R^{\Sigma}(w)$ (where $R_{F}=\bigcup_{\varphi \in \mathrm{S}(\mathcal{L}(\boxminus,>)} F(w,[\varphi])$ ): Suppose $v \in R_{F}(w)$, then there is $\theta$ such that $\{\psi: \theta>\psi \in w\} \subseteq v$. But $\{\varphi: \neg \varphi>$ $\varphi \in w\} \subseteq\{\psi: \theta>\psi \in w\}$ : Let $\varphi$ be in the first set. Then $\neg \varphi>\varphi \in w$ thus $\theta>\varphi \in w$ (MOD, cf. Lemma 7.5) for any $\theta$, thus $\varphi \in\{\psi: \theta>\psi \in w\}$. Thereby $\{\varphi: \square \varphi \in w\} \subseteq v$, i.e., $\{\varphi: \boxminus \varphi \in w\} \subseteq v$ (IA). Hence $\langle w, v\rangle \in R^{\Sigma}$. Then restrict the model with equivalence relation $R^{\Sigma}$ for $\boxminus$ to an absolute equivalence relation (cf. proof of Corollary 3.8 for regular ranked models, p. 881). The transfer to absolute ranked models goes as before.

LEMMA 7.14. The doxastic conditional $>$ is covariantly definable in the absolute ranking semantics for $\mathcal{L}_{>, \boxminus}$ by $(\psi \triangleright \chi)^{*}:=\left(\left(\psi^{*}>\chi^{*}\right) \wedge \neg\left(\psi^{*}>\perp\right)\right) \vee\left(\boxminus \chi^{*} \wedge\left(\psi^{*}>\right.\right.$ $\perp)$ ).

Proof. To a ranked model $\mathfrak{R}$ associate $\mathfrak{R}^{*}=\langle\mathfrak{R}, R\rangle$, where $R=W^{2}$ defines $\boxminus$. Covariance by induction. For $(\psi \triangleright \chi)^{*}$ consider the (induced) selection function $F$ in $\mathfrak{R}^{*}$ and $G$ generating the conditional clause in the doxastic model $\mathfrak{R}$. Then:

$$
G(w, A)= \begin{cases}F(w, A) & \text { if } F(w, A) \neq \emptyset \\ W & \text { otherwise }\end{cases}
$$

Thus

$$
\begin{array}{ll} 
& w, \mathfrak{R} F(\psi \triangleright \chi) \\
\text { iff } & G(w,[\psi]) \subseteq[\chi] \\
\text { iff } & G\left(w,\left[\psi^{*}\right]\right) \subseteq\left[\chi^{*}\right] \\
\text { iff } & \left(\emptyset \neq F\left(w,\left[\psi^{*}\right]\right) \subseteq\left[\chi^{*}\right]\right) \text { or }\left(\left[\chi^{*}\right]=\left[\top^{*}\right] \& F\left(w,\left[\psi^{*}\right]\right)=\emptyset\right) \\
\text { iff } & w, \mathfrak{R}^{*} \vDash\left(\left(\psi^{*}>\chi^{*}\right) \wedge \neg\left(\psi^{*}>\perp\right)\right) \vee\left(\boxminus \chi^{*} \wedge\left(\psi^{*}>\perp\right)\right) \\
\text { iff } & w, \mathfrak{R}^{*} \vDash(\psi \triangleright \chi)^{*}
\end{array}
$$

LEMMA 7.15. In the absolute ranking semantics $\models^{*}$ :

$$
\text { 1. }(\varphi \triangleright \varphi)^{*} \equiv \neg\left(\varphi^{*}>\perp\right)\left[=\diamond \varphi^{*}\right] . \quad \text { 2. } \boxminus \psi^{*} \equiv(\perp \triangleright \psi)^{*}\left[\equiv(\neg \psi \triangleright \psi)^{*}\right] .
$$

Proof.

(1) Suppose $\neg\left(\varphi^{*}>\perp\right) . \varphi^{*}>\varphi^{*}$ (ID) implies $(\varphi>\varphi)^{*}$. Suppose $(\varphi>\varphi)^{*}$, i.e., $\left(\varphi^{*}>\right.$ $\left.\varphi^{*} \wedge \neg\left(\varphi^{*}>\perp\right)\right)$ or $\left(\boxminus \varphi^{*} \wedge\left(\varphi^{*}>\perp\right)\right)$. The first implies $\neg\left(\varphi^{*}>\perp\right)$. The second is contradictory, since $\boxminus \varphi \rightarrow \square \varphi$ (IA), $\square \varphi \rightarrow \diamond \varphi$ (D by P), but $\nabla \varphi^{*}=\neg\left(\varphi^{*}>\perp\right)$ contradicts $\varphi^{*}>\perp$.

(2)

$$
\begin{aligned}
& (\perp \triangleright \psi)^{*}=\left(\left(\perp>\psi^{*}\right) \wedge \neg(\perp>\perp)\right) \vee\left(\boxminus \psi^{*} \wedge(\perp>\perp)\right) \\
& \equiv\left(\left(\perp>\psi^{*}\right) \wedge \perp\right) \vee\left(\boxminus \psi^{*} \wedge \top\right) \\
& \equiv \boxminus \psi^{*} \\
& (\neg \psi \triangleright \psi)^{*} \\
& =\left(\left(\neg \psi^{*}>\psi^{*}\right) \wedge \neg\left(\neg \psi^{*}>\perp\right)\right) \vee\left(\boxminus \psi^{*} \wedge\left(\neg \psi^{*}>\perp\right)\right) \\
& \equiv \perp \vee\left(\boxminus \psi^{*} \wedge \square \psi^{*}\right) \\
& \equiv \boxminus \psi^{*}
\end{aligned}
$$


DEFINITION 7.16. A conditional $\longrightarrow$ and a two-place operator $O$ are antecedent disjunctive (resp., cross disjunctive), iff $(\varphi \triangleright \psi) \wedge O[\varphi, \chi]($ resp. $\varphi \triangleright \psi \wedge O[\psi, \varphi])$ is inconsistent.

LEMMA 7.17. In the absolute ranking semantics and for $\varphi>\psi=((\varphi>\psi) \wedge \neg(\varphi>$ $\perp))$ and $O[\varphi, \psi]=\boxminus \psi \wedge(\varphi>\perp):(1) \triangleright, O$ are antecedent and cross disjunctive, (2) $O$ validates $\mathrm{RCEA}$ and $\mathrm{CC}$; and $(\mathrm{CO}): O[\varphi, \psi] \rightarrow((\chi>\psi) \vee O[\chi, \psi])$.

Proof. (1) $(\varphi>\psi) \wedge \neg(\varphi>\perp)$ contradicts $\boxminus \chi \wedge(\varphi>\perp)$. And $(\varphi>\psi) \wedge \neg(\varphi>$ $\perp)$ contradicts $\boxminus \varphi \wedge(\psi>\perp): \psi>\perp$ implies $\psi>\varphi(\mathrm{RCM})$. But since $\varphi>\psi$ : $\varphi>\perp$ (CSO, cf. Lemma 7.6). Contradiction. (2) RCEA $O: \boxminus, \square$ satisfy SLE (RCEA, $>$ ). CC $O$ : $\boxminus$ is closed under conjunction. CO: Suppose $(\boxminus \psi \wedge \varphi>\perp)$. $\boxminus \psi$ implies $\square \psi$ (IA), i.e., $\neg \psi>\perp$ thus $\neg \psi>\psi$ (RCM). Hence (MOD, cf. Lemma 7.5) $\chi>\psi$. But $(\chi>\perp) \vee \neg(\chi>\perp) . \neg(\chi>\perp)$ and $\chi>\psi$ yields $(\chi>\psi) . \chi>\perp$ and $\boxminus \psi$ yields $O[\chi, \psi]$.

THEOREM 7.18. AXd (see p. 872) is sound for the doxastic ranking semantics.

Proof. Using the sound axiomatisation of the neutral conditional $>$ (Theorem 7.10) within the absolute ranking semantics $\vDash^{*}$, covariance of $\triangleright$ (Lemma 7.14), and Lemma 7.17. MoPo, PT are clear.

RCEA Suppose $\mathfrak{R} \vDash \varphi \leftrightarrow \psi, \varphi \triangleright \chi$. Thus $\mathfrak{R}^{*} \vDash^{*} \varphi^{*} \leftrightarrow \psi^{*},\left(\varphi^{*}>\chi^{*}\right) \vee O\left[\varphi^{*}, \chi^{*}\right]$ (covariance). Hence $\mathfrak{R}^{*} \models^{*}\left(\psi^{*} \triangleright \chi^{*}\right) \vee O\left[\psi^{*}, \chi^{*}\right]$ (RCEA: $\left.\rightarrow, O\right)$. RCM similarly.

CC Suppose $w, \mathfrak{R} \vDash \varphi \triangleright \psi, \varphi \triangleright \chi$. Thus $w, \mathfrak{R}^{*} \vDash\left(\varphi^{*} \triangleright \psi^{*}\right) \vee O\left[\varphi^{*}, \psi^{*}\right],\left(\varphi^{*} \triangleright \chi^{*}\right) \vee$ $O\left[\varphi^{*}, \chi^{*}\right]$. This yields 4 cases (coding see footnote 66): $11 \varphi^{*}>\psi^{*}, \varphi^{*}>\chi^{*} ; 12$ $\varphi^{*} \psi^{*}, O\left[\varphi^{*}, \chi^{*}\right] ; 21 O\left[\varphi^{*}, \psi^{*}\right], \varphi^{*} \chi^{*}$ or $22 O\left[\varphi^{*}, \psi^{*}\right], O\left[\varphi^{*}, \chi^{*}\right] .11,22$ yield $\varphi^{*} \triangleright\left(\psi^{*} \wedge \chi^{*}\right)$, resp. $O\left[\varphi^{*}, \psi^{*} \wedge \chi^{*}\right](\mathrm{CC}: \triangleright, \mathrm{O}) .12,21$ are inconsistent (antecedentdisjuntive - cf. Lemma 7.17).

CV, CA, CMon, C Similarly, using, (CO) for $O$ (Lemma 7.17). Con, CN, A are obvious.

B Suppose: $\varphi \triangleright \chi, \neg(\psi \triangleright \psi)$ and $\neg(\perp \triangleright \chi) . \varphi \triangleright \chi$ has 2 or-clauses. But $\neg(\perp \triangleright \chi)$, yielding $\neg \boxminus \chi^{*}$, excludes the second. Thus $\left(\varphi^{*}>\chi^{*}\right) \wedge \neg\left(\varphi^{*}>\perp\right) . \neg(\psi \triangleright \psi)$ implies $\psi^{*}>\perp$ (Lemma 7.15). Thus $\psi^{*}>\chi^{*}(\mathrm{RCM})$ and hence (i) $\left(\varphi^{*} \vee \psi^{*}\right)>\chi^{*}(\mathrm{CA})$ and (ii) $\neg\left(\left(\varphi^{*} \vee \psi^{*}\right)>\perp\right)$ [otherwise $\left(\varphi^{*} \vee \psi^{*}\right)>\varphi^{*}(\mathrm{RCM})$ and thus $\varphi^{*}>\perp(\mathrm{CMon})-$ contradiction]. Therefore (i, ii) $(\varphi \vee \psi) \triangleright \chi$.

E, F Similar to B, using Lemma 7.15. K*, T*, 5* using K, T, 5 for $\boxminus$.

Define the translation $\bullet: \mathrm{S}\left(\mathcal{L}_{>, \square}\right) \longrightarrow \mathrm{S}\left(\mathcal{L}_{\triangleright}\right)$ by $p^{\bullet}:=p,(\neg \psi)^{\bullet}:=\neg \psi^{\bullet},(\psi \circ \chi)^{\bullet}:=$ $\left(\psi^{\bullet} \circ \chi^{\bullet}\right),(\boxminus \varphi)^{\bullet}:=\perp \triangleright \varphi^{\bullet}$, and $(\varphi>\psi)^{\bullet}:=\left(\varphi^{\bullet} \triangleright \psi^{\bullet}\right) \vee\left(\neg\left(\varphi^{\bullet} \triangleright \varphi^{\bullet}\right) \wedge \neg\left(\perp \triangleright \psi^{\bullet}\right)\right)$.

LEMMA 7.19. The translations are inverses to each other:
1. For every $\varphi \in \mathrm{S}\left(\mathcal{L}_{\triangleright}\right),\left(\varphi^{*}\right)^{\bullet}=\varphi$,
2. For every $\varphi \in \mathrm{S}\left(\mathcal{L}_{>}, \boxminus\right),\left(\varphi^{\bullet}\right)^{*}=\varphi$.

Proof. (1.) Consider $\varphi \triangleright \psi(7.15$ refers to Lemma 7.15):

$$
\begin{aligned}
& \left((\varphi \triangleright \psi)^{*}\right)^{\bullet} \\
= & \left(\left(\left(\varphi^{*}>\psi^{*}\right)^{\bullet} \wedge \neg\left(\varphi^{*}>\perp\right)^{\bullet}\right) \vee\left(\left(\boxminus \psi^{*}\right)^{\bullet} \wedge\left(\varphi^{*}>\perp\right)^{\bullet}\right)\right) \\
= & \left(\left(\left(\varphi^{* \bullet} \triangleright \psi^{* \bullet}\right) \vee\left(\neg\left(\varphi^{* \bullet} \triangleright \varphi^{* \bullet}\right) \wedge \neg \boxminus \psi^{* \bullet}\right)\right) \wedge\left(\varphi^{* \bullet} \triangleright \varphi^{* \bullet}\right)\right) \\
& \left.\vee\left(\left(\perp \triangleright \psi^{* \bullet}\right) \wedge \neg\left(\varphi^{* \bullet} \triangleright \varphi^{* \bullet}\right)\right)\right) \\
= & (((\varphi \triangleright \psi) \vee(\neg(\varphi \triangleright \varphi) \wedge \neg \boxminus \psi)) \wedge(\varphi \triangleright \varphi)) \vee((\perp \triangleright \psi) \wedge \neg(\varphi \triangleright \varphi))) \\
= & ((\varphi \triangleright \psi) \wedge(\varphi \triangleright \varphi)) \vee \perp \vee((\perp \triangleright \psi) \wedge \neg(\varphi \triangleright \varphi)) \\
= & ((\varphi \triangleright \psi) \wedge(\varphi \triangleright \varphi)) \vee((\varphi \triangleright \psi) \wedge \neg(\varphi \triangleright \varphi)) \\
= & \varphi \triangleright \psi
\end{aligned}
$$


(2.) Similarly.

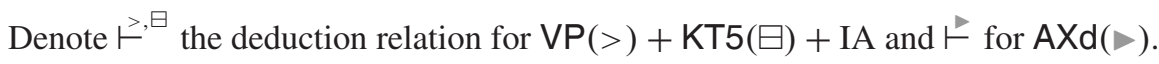

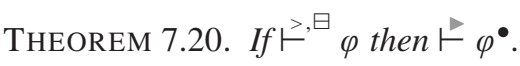

Proof. By induction on the length $n$ of the proof (cf. Theorem 7.11):

$n=1$ : Then $\theta$ is PT, K, T, 5, IA, ID, CC, CA, CMon, $\mathbf{C V}$, or $\mathbf{P}$ from $\mathrm{AX}=\mathrm{VP}(>)+$ $\mathrm{KNT5}(\boxminus)+\mathrm{IA})$. Then $\theta^{\bullet}$ is provable in $\mathrm{AX} \bullet=\mathrm{AXd}$ : PT from $\mathrm{PT}(\triangleright), \mathrm{KT} 5(\triangleright)$ are axioms. $\mathbf{P}$ from $\mathrm{T}(\triangleright)$. For ID use C, for $\mathbf{C C}$ use $\mathrm{CC}(\triangleright)$ and $\mathrm{RCM}(\triangleright)$, for $\mathbf{C A}$ use $\mathrm{CA}(\triangleright), \mathrm{B}$ and $\mathrm{E}$, for $\mathbf{C V}$ use $\mathrm{CV}(\triangleright)$ and F. Eg.:

IA Suppose $\theta=\boxminus \varphi \rightarrow(\neg \varphi>\perp)$. Assume (i) $\perp \triangleright \varphi^{\bullet}$. We show $\left(\neg \varphi^{\bullet} \triangleright \perp\right) \vee$ $\left(\neg\left(\neg \varphi^{\bullet} \triangleright \neg \varphi^{\bullet}\right) \wedge \neg(\perp \triangleright \perp)\right)$. (ii) $\neg(\perp \triangleright \perp)$ by Con. From (i) we obtain $\neg \varphi^{\bullet} \triangleright \varphi^{\bullet}$ (C). Suppose (for reductio) $\neg \varphi^{\bullet} \triangleright \neg \varphi^{\bullet}$. Then $\neg \varphi^{\bullet} \triangleright \perp$ (CC), contradicting (Con). Thus (iii) $\neg\left(\neg \varphi^{\bullet} \triangleright \neg \varphi^{\bullet}\right)$. (ii, iii) yield $(\neg \varphi>\perp)^{\bullet}$.

CMon Let $\theta=((\varphi>\chi) \wedge(\varphi>\psi)) \rightarrow((\varphi \wedge \psi)>\chi)$. Assume $\left(\left(\varphi^{\bullet} \triangleright \chi^{\bullet}\right) \vee\right.$ $\left.\left(\neg\left(\varphi^{\bullet} \triangleright \varphi^{\bullet}\right) \wedge \neg\left(\perp \triangleright \chi^{\bullet}\right)\right)\right) \wedge\left(\left(\varphi^{\bullet} \triangleright \psi^{\bullet}\right) \vee\left(\neg\left(\varphi^{\bullet} \triangleright \varphi^{\bullet}\right) \wedge \neg\left(\perp \triangleright \psi^{\bullet}\right)\right)\right)$. This yields 4 cases (coding see footnote 66). 11 yields $\left(\varphi^{\bullet} \wedge \psi^{\bullet}\right) \triangleright \chi^{\bullet}($ CMon $\triangleright) .12$ yields $\varphi^{\bullet} \triangleright \chi^{\bullet}$, $\neg\left(\varphi^{\bullet} \triangleright \varphi^{\bullet}\right)$ and $\neg\left(\perp \triangleright \psi^{\bullet}\right)$. The first two imply $\perp \triangleright \chi^{\bullet}(\mathrm{A})$ thus $\left(\varphi^{\bullet} \wedge \psi^{\bullet}\right) \triangleright \chi^{\bullet}(\mathrm{C}) .21$ yields $\varphi^{\bullet} \triangleright \psi^{\bullet}, \neg\left(\varphi^{\bullet} \triangleright \varphi^{\bullet}\right)$ and $\neg\left(\perp \triangleright \chi^{\bullet}\right)$. The second yields $\neg\left(\left(\varphi^{\bullet} \wedge \psi^{\bullet}\right) \triangleright\left(\varphi^{\bullet} \wedge \psi^{\bullet}\right)\right)$ $(\mathrm{F})$, and hence $\neg\left(\left(\varphi^{\bullet} \wedge \psi^{\bullet}\right) \triangleright\left(\varphi^{\bullet} \wedge \psi^{\bullet}\right)\right) \wedge \neg\left(\perp \triangleright \chi^{\bullet}\right) .22$ yields $\neg\left(\perp \triangleright \chi^{\bullet}\right), \neg\left(\perp \triangleright \psi^{\bullet}\right)$ and $\neg\left(\varphi^{\bullet} \triangleright \varphi^{\bullet}\right)$, and one proceeds as in 21 .

$n+1$. Cf. Theorem 7.11. Necessitation $\mathbf{N}$ from (CN, RCM), RCM similar to RCEA (using $\operatorname{RCM}(\triangleright)$ and $\mathrm{C})$, eg.:

RCEA Let $\theta_{n+1}=\psi>\chi$ and there are $\theta_{i}=\varphi \leftrightarrow \psi$ and $\theta_{j}=\varphi>\chi(i, j \leq n)$ and the property holds for $i, j$. By (IH) we have a proof of $\theta_{i}^{\bullet}=\varphi^{\bullet} \leftrightarrow \psi^{\bullet}$ and of $\theta_{j}^{\bullet}=$ $(\varphi>\chi)^{\bullet}=\left(\left(\varphi^{\bullet} \triangleright \chi^{\bullet}\right) \vee\left(\neg\left(\varphi^{\bullet} \triangleright \varphi^{\bullet}\right) \wedge \neg\left(\perp \triangleright \psi^{\bullet}\right)\right)\right)$ in AXं. Assume $\varphi^{\bullet} \triangleright \chi^{\bullet}$. Thus $\psi^{\bullet} \triangleright \chi^{\bullet}($ RCEA $)$. Assume $\left(\neg\left(\varphi^{\bullet} \triangleright \varphi^{\bullet}\right) \wedge \neg\left(\perp \triangleright \chi^{\bullet}\right)\right)$. Thus $\left(\neg\left(\psi^{\bullet} \triangleright \psi^{\bullet}\right) \wedge \neg\left(\perp \triangleright \chi^{\bullet}\right)\right)$ (RCEA). Therefore $\theta_{n+1}^{\bullet}=(\psi>\chi)^{\bullet}$ in both cases.

\section{COROLlaRY 7.21. AXd is complete for the doxastic ranking semantics.}

Proof. Suppose $\nvdash^{\not} \varphi$. Then $\nvdash^{\not}\left(\varphi^{*}\right)^{\bullet}$ (Lemma 7.19). Thus $\not{\nvdash}^{,} \varphi^{*}$ (Theorem 7.20). Hence there is an absolute ranked model $\mathfrak{R}^{*}$, where $\mathfrak{R}^{*} \not \nvdash^{*} \varphi^{*}$ (completeness, Theorem 7.13). Yet, the underlying ranked model $\mathfrak{R}$ of $\mathfrak{R}^{*}$, with the doxastic semantics, covaries in truth (Lemma 7.14). Thus $\mathfrak{R \not} \varphi$. Hence $\not{\not} \varphi$.

7.4.3. Metaphysical Conditional. Denote the metaphysical truth relation by $\Vdash$. To a ranked model $\mathfrak{R}$ associate the absolute ranked model $\mathfrak{R}^{*}$ with truth relation $\stackrel{*}{*}^{\text {(see }}$ p. 883).

LEMMA 7.22. The metaphysical conditional is covariantly definable in $\vDash^{*}$ by $(\varphi \triangleright \psi)^{*} \equiv\left(\varphi^{*}>\psi^{*} \wedge \neg\left(\varphi^{*}>\perp\right)\right) \vee\left(\boxminus\left(\varphi^{*} \rightarrow \psi^{*}\right) \wedge\left(\varphi^{*}>\perp\right)\right)$.

Proof. By induction on the complexity of the formula. Cf. Lemma 7.14.

LEMMA 7.23. In the absolute ranking semantics and for $\varphi>\psi=((\varphi>\psi) \wedge \neg(\varphi>$ $\perp)$ ) and $O[\varphi, \psi]=\boxminus(\varphi \rightarrow \psi) \wedge(\varphi>\perp)$. (1) $O$ validates RCEA, RCM, CC, CMon, CA, CV and (2) $\downarrow, O$ are antecedent and cross disjunctive and (3) validate

- $((\varphi \triangleright \chi) \wedge O[\psi, \chi]) \rightarrow((\varphi \vee \psi) \triangleright \chi)$ 
Proof.

(1) RCEA, RCM, CC, CMon, CA: Since the strict conditional and > validate these. CV: Suppose $\boxminus(\varphi \rightarrow \chi) \wedge(\varphi>\perp)$ and $\neg \boxminus(\varphi \rightarrow \psi)$ or $\neg(\varphi>\perp)$. The second combination is inconsistent. The first yields $\boxminus((\varphi \wedge \psi) \rightarrow \chi)(\mathrm{CV}$, strict conditional). And $\varphi>\perp$ implies $(\varphi \wedge \psi)>\perp$ (CMon, RCM).

(2) as (1) in Lemma 7.17.

(3) Suppose $\varphi>\chi, \neg(\varphi>\perp), \boxminus(\psi \rightarrow \chi), \psi>\perp$. Thus $(\varphi \vee \psi)>\chi$ (RCM, CA). Additionally $\neg((\varphi \vee \psi)>\perp)$, since $\neg(\varphi>\perp)$.

THEOREM 7.24. VT5 is sound for $\Vdash$.

Proof. Using the sound axiomatisation of the neutral conditional $\downarrow$ (Theorem 7.10), covariance $-($ Lemma 7.22) and Lemma 7.23 (cf. Theorem 7.18). MoPo, PT, RCEA, RCM, CC work similarly. P from T. For CMon, CV, CA, use Lemma 7.23. Eg.:

CMon Suppose $w, \mathfrak{R} \vDash \varphi \triangleright \chi, \varphi \triangleright \psi$. Thus (covariance) $w, \mathfrak{R}^{*} \vDash^{*}(\varphi \triangleright \chi)^{*} \vee$ $O\left[\varphi^{*}, \chi^{*}\right],(\varphi \triangleright \psi)^{*} \vee O\left[\varphi^{*}, \psi^{*}\right] .11^{67}$ yields $(\varphi \wedge \psi) \triangleright \chi($ CMon: $\vee) .12$, 21 are inconsistent (antecedent-disjuntive, Lemma 7.23.2). 22 yields $O\left[\varphi^{*}, \chi^{*}\right]$ and $O\left[\varphi^{*}, \psi^{*}\right]$ thus $O\left[\varphi^{*} \wedge \psi^{*}, \chi^{*}\right]$ (CMon, $O$ ).

$\mathrm{CV}$ Case 1 by $\mathrm{CV}(\triangleright)$. Case $2 O\left[\varphi^{*}, \chi^{*}\right], \neg\left(\varphi^{*} \neg \neg \psi^{*}\right) \wedge \neg O\left[\varphi^{*}, \neg \psi^{*}\right]$ yields $O\left[\varphi^{*} \wedge \psi^{*}, \chi^{*}\right](\mathrm{CV}, O)$.

CA Case 11 by CA $(\triangleright)$. Case $12 \varphi^{*} \triangleright \chi^{*}, O\left[\psi^{*}, \chi^{*}\right]$ yields $\left(\varphi^{*} \vee \psi^{*}\right) \triangleright \chi^{*}($ crossCA). 21 similarly. $22 O\left[\varphi^{*}, \chi^{*}\right], O\left[\psi^{*}, \chi^{*}\right]$ yields $O\left[\varphi^{*} \vee \psi^{*}, \chi^{*}\right]$ by (CA, $O)$.

ID We have $\varphi>\varphi$ as well as $\boxminus(\varphi \rightarrow \varphi)$. Hence $\varphi \triangleright \varphi$, since $(\varphi>\perp) \vee \neg(\varphi>\perp)$.

T Suppose $\neg \varphi \triangleright \perp$. Thus (1) $\left(\neg \varphi^{*}>\perp\right) \wedge \neg\left(\neg \varphi^{*}>\perp\right)$ or (2) $\boxminus\left(\neg \varphi^{*} \rightarrow\right.$ $\perp) \wedge\left(\neg \varphi^{*}>\perp\right)$. (1) is inconsistent and (2) reduces to $\boxminus \varphi^{*}(\mathrm{IA}, \boxminus)$. But then $\varphi^{*}(\mathrm{~T}, \boxminus)$, i.e., $\varphi$ (covariance).

$5 \boxminus \varphi \equiv \square_{\triangleright} \varphi=: \neg \varphi \triangleright \perp$ (Lemma 7.25.1).

Suppose $\neg(\varphi \triangleright \perp)$. Thus $\neg\left(\left(\varphi^{*}>\perp\right) \wedge \neg\left(\varphi^{*}>\perp\right)\right)$ (a tautology) and $\neg\left(\boxminus\left(\varphi^{*} \rightarrow \perp\right) \wedge\left(\varphi^{*}>\perp\right)\right)$. Therefore $(1) \neg \boxminus\left(\varphi^{*} \rightarrow \perp\right)$ or $(2) \neg\left(\varphi^{*}>\perp\right)$. (1) implies $\diamond \varphi^{*}$ and thus $\boxminus \diamond \varphi^{*}(5, \boxminus)$, establishing 5 for $\triangleright$, since $\boxminus \varphi \equiv$ $\left(\square_{\triangleright} \varphi\right)^{*}=:(\neg \varphi \triangleright \perp)^{*}\left(\right.$ Lemma 7.25) (2) implies $\diamond \varphi^{*}$ and thus $\forall \varphi^{*}$ (IA), establishing 5 for $\triangleright$.

LEMMA 7.25. In the absolute ranking semantics: $\boxminus \varphi \equiv(\neg \varphi \triangleright \perp)^{*}$.

Proof. $\boxminus(\varphi \rightarrow \perp)$ implies $\varphi>\perp$ (IA). Therefore $((\varphi>\perp) \wedge \neg(\varphi>\perp)) \vee(\boxminus(\varphi \rightarrow$ $\perp) \wedge(\varphi>\perp)) \equiv(\boxminus(\varphi \rightarrow \perp) \wedge(\varphi>\perp)) \equiv \boxminus(\varphi \rightarrow \perp) \equiv \boxminus \neg \varphi$.

But $\varphi>\psi$ is not definable by $\triangleright$. Thus we adopt another strategy for completeness, using the finite model property of VT5. Let $\mathfrak{R}=\left\langle W,\left(\kappa^{w}\right)_{w \in W}, V\right\rangle$ be a finite ranked model. Thus there is $N \in \mathbb{N}$, such that for all $n \in \mathbb{N}$ and all $w, v \in W$, if $\kappa^{w}(v)<\infty$ then $\kappa^{w}(v)<N$. Hence we may regularise $\mathfrak{R}$ by

$$
\rho^{w}(v)= \begin{cases}\kappa^{w}(v) & \text { if } \kappa^{w}(v)<\infty \\ N & \text { otherwise }\end{cases}
$$

67 Coding see footnote 66. 
Denote $\mathfrak{R}^{+}=\left\langle W,\left(\rho^{w}\right)_{w \in W}, V\right\rangle$ the regularisation of the above $\mathfrak{R}, \models^{+}$its standard truth relation. Consider the trivial translation $p^{+}=p,(\neg \psi)^{+}=\neg \psi^{+},(\psi \circ \chi)^{+}=\psi^{+}$。 $\chi^{+},(\psi \triangleright \chi)^{+}=\psi^{+}>\chi^{+}$, which we may ignore:

LEMMA 7.26. If $\mathfrak{R}$ is a finite ranked model, then for all $\varphi \in \mathrm{S}\left(\mathcal{L}_{>}\right)$and all $w \in W$, $w, \mathfrak{R} \vDash \varphi$ iff $w, \mathfrak{R}^{+} \vDash^{+} \varphi$.

Proof. Clear.

COROLLARY 7.27. VT5 is sound and complete for the metaphysical ranking semantics.

Proof. Soundness: Theorem 7.24. Completeness: Suppose $\vDash \varphi$. Then this holds for finite metaphysical ranked models, especially for regular ones. Thus $\models^{+} \varphi$ for finite regular ranked models (Lemma 7.26). Thus $\operatorname{VTT}_{5} \varphi$ (by the finite model property).

7.4.4. Alternative Conditional. Proof of Theorem 5.4. Let $\Vdash$ be the metaphysical truth relation, $\models^{*}$ the alternative truth relation and $\vDash$ the standard truth relation. For every ranked model $\mathfrak{R}=\left\langle W,\left(\kappa^{w}\right)_{w \in W}, V\right\rangle$, there is a squeezed model $\mathfrak{R}^{s}=\left\langle W,\left(\lambda^{w}\right)_{w \in W}, V\right\rangle$, where $\lambda^{w}(v):=\kappa^{w}(v)$ if $\kappa^{w}(v)>\kappa^{w}(w)$ and $\lambda^{w}(v):=0$ otherwise. The squeezed model is a weakly centered $(\kappa \mathrm{cw})$ ranked model, since $\lambda^{w}(w):=0$. Additionally $\vDash_{\mathfrak{R}^{s}}$ is faithful to $\models_{\mathfrak{R}}^{*}$. This is clear for nonconditional sentences. Now consider $\varphi>\psi$ and assume the property for $\varphi, \psi$, so that we can write $[\varphi]=[\varphi]^{F_{\mathfrak{R}}^{*}}=[\varphi]^{\vdash_{\mathfrak{R}}^{s}}$ and $[\psi]=[\psi]^{F_{\mathfrak{R}}^{*}}=$ $[\psi]^{\vee_{R^{s}}}$, and abbreviate $\kappa^{w}(\varphi)=\kappa^{w}\left([\varphi]^{F_{\mathfrak{R}}^{*}}\right)$ and $\lambda^{w}(\varphi)=\lambda^{w}\left([\varphi]^{\vee_{\mathfrak{R}^{s}}}\right)$ etc. Let $\lambda_{\varphi}^{w}$ denote the metaphysical conditionalisation of $\lambda^{w}$. Then

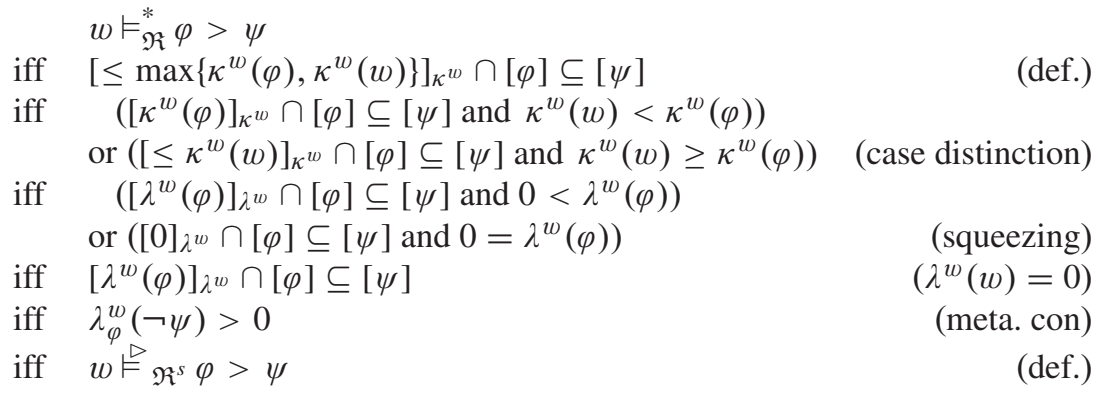

Soundness: Suppose $\not \nvdash^{*} \varphi$. Thus there is a ranked model $\mathfrak{R}=\left\langle W,\left(\kappa^{w}\right)_{w \in W}, V\right\rangle$ and $w \in W$ such that $w \nvdash_{\mathfrak{R}}^{*} \varphi$. Hence (by faithfulness) $w \not_{\mathfrak{R}^{s}} \varphi$ holds for the squeezing $\mathfrak{R}^{s}$ of $\mathfrak{R}$. But $\mathfrak{R}^{s}$ is a cw-ranked model and thus $\not^{\prime} \varphi$ holds in the cw-metaphysical ranking semantics. Therefore $\nvdash$ VWT5 $\varphi$ (completeness for VWT5 w.r.t. the cw-metaphysical ranking semantics), hence $\nvdash_{\mathrm{VW}}$ (VW5 is equivalent to VWT5).

Completeness: Suppose $\nvdash_{\text {VW5 }} \varphi$. Hence $\nvdash_{\text {VWT5 }} \varphi$. Thus there is a regular cw-ranked model $\mathfrak{R}$ and $w \in W$ such that $w \nvdash_{\mathfrak{R}} \varphi$ (completeness for regular cw-ranked models). But for a regular cw-ranked model $\mathfrak{R}, \models_{\mathfrak{R}}$ and $\models_{\mathfrak{R}}^{*}$ agree (since $\max \left\{\kappa^{w}(w), \kappa^{w}(A)\right\}=\kappa^{w}(A)$ and $\kappa^{w}(A)=\infty$ only for $\left.A=\emptyset\right)$. Thus $w \nvdash_{\mathfrak{R}}^{*} \varphi$. Therefore $\not \nvdash^{*} \varphi$ in the alternative ranking semantics.

Proof of Theorem 5.5. Let $\vDash$ the standard truth definition (1) and $\vDash^{*}$ the alternative standard truth definition (1A). Let $\mathfrak{R}=\left\langle W,\left(\kappa^{w}\right)_{w \in W}, V\right\rangle$ be a ranked model. Define the 
selection model $\mathfrak{M}=\langle W, F, V\rangle$, where

$$
F(w, A):= \begin{cases}{\left[\leq \max \left\{\kappa^{w}(w), \kappa^{w}(A)\right\}\right]_{\kappa^{w}} \cap A} & \text { if } \kappa^{w}(A)<\infty, \\ \varnothing & \text { if } \kappa^{w}(A)=\infty .\end{cases}
$$

Note: if $\kappa^{w}(w)=\infty$ and $\kappa^{w}(A)<\infty$, we obtain $F(w, A)=A$ ! One verifies that this is a selection function which is $\mathrm{p}$, id, ca, cmon, $\mathrm{cv}$ and additionally $\mathrm{mp}^{\diamond}$ : If $F(w, A) \neq \varnothing$ then $(F(w, A) \subseteq B$ implies $w \in \bar{A} \cup B)$. Indeed $F(w, A) \neq \varnothing \operatorname{implies} \kappa^{w}(A)<\infty$. $F(w,$.$) will then be weakly centred, since F(w, A)=\left[\leq \max \left\{\kappa^{w}(w), \kappa^{w}(A)\right\}\right]_{\kappa^{w}} \cap A=$ $\left[\leq \lambda^{w}(A)\right]_{\lambda^{w}} \cap A$, for the squeezing $\lambda^{w}$ of $\kappa^{w}$, since $\lambda^{w}$ is weakly centred (compare the proof of Theorem 5.4).

Additionally $\mathfrak{M} \vDash$ is faithful to $\mathfrak{R} \models^{*}$ (by induction). Consider the case $\varphi>\psi$ and assume the property for $\varphi, \psi(\mathrm{IH})$. Then

$$
\begin{array}{llr} 
& w \vDash_{\mathfrak{R}}^{*} \varphi>\psi & \\
\text { iff } & \kappa_{[\varphi]^{\mathfrak{R}}}^{w}\left([\neg \psi]^{\mathfrak{R}}\right)>\max \left\{0, \kappa^{w}(w)-\kappa^{w}\left([\varphi]^{\mathfrak{R}}\right)\right\} \text { or } \kappa^{w}\left([\varphi]^{\mathfrak{R}}\right)=\infty & \text { (def. } \left.\vDash_{\mathfrak{R}}^{*}\right) \\
\text { iff } & \left(\left[\leq \max \left\{\kappa^{w}\left([\varphi]^{\mathfrak{R}}\right), \kappa^{w}(w)\right\}\right]_{\kappa^{w}} \cap[\varphi]^{\mathfrak{R}} \subseteq[\psi]^{\mathfrak{R}} \text { and } \kappa^{w}\left([\varphi]^{\mathfrak{R}}\right)<\infty\right) & \\
& \text { or } \kappa^{w}\left([\varphi]^{\mathfrak{R}}\right)=\infty & \text { (Transformation) } \\
\text { iff } & F\left(w,[\varphi]^{\mathfrak{R}}\right) \subseteq[\psi]^{\mathfrak{R}} \text { or } F\left(w,[\varphi]^{\mathfrak{R}}\right)=\emptyset & \text { (def. } F \text { ) } \\
\text { iff } & F\left(w,[\varphi]^{\mathfrak{R}}\right) \subseteq[\psi]^{\mathfrak{R}} & \text { (Logic) } \\
\text { iff } & F\left(w,[\varphi]^{\mathfrak{M}}\right) \subseteq[\psi]^{\mathfrak{M}} & \text { (IH) } \\
\text { iff } & w \vDash_{\mathfrak{M}} \varphi>\psi & \text { (def. } \vDash_{\mathfrak{M})}
\end{array}
$$

Soundness: Suppose $\not^{*} \varphi$. Thus there is a ranked model $\mathfrak{R}$ and $w \in W$ such that $w \nvdash_{\mathfrak{R}}^{*} \varphi$. Hence for the corresponding selection model $\mathfrak{M}, w \nvdash_{\mathfrak{M}} \varphi$ (faithfulness). But $\mathfrak{M}$ is an $\left(\mathrm{mp}^{\diamond}\right)$-ranky selection model and thus $\not \models \varphi$ in the $\left(\mathrm{mp}^{\diamond}\right)$-ranky selection semantics. Therefore $\nvdash_{\mathrm{VP}+\mathrm{MP}} \diamond \varphi$ (one shows: completeness for $\mathrm{VP}+\mathrm{MP}^{\diamond}$ w.r.t. the (mp $\left.{ }^{\diamond}\right)$-ranky selection semantics as in Corollary 7.4).

Completeness: Suppose $\nvdash_{\mathrm{VP}+\mathrm{MP}} \diamond \varphi$. Thus there is an (mp ${ }^{\diamond}$ )-ranky selection model $\mathfrak{M}$ and $w \in W$ such that $w \nvdash_{\mathfrak{M}} \varphi$. Through $\varphi$-filtration we obtain a $\left(\kappa \mathrm{mp} \nabla^{\diamond}\right)$-ranked model $\mathfrak{R}$ which is faithful, i.e., $w \nvdash_{\mathfrak{R}} \varphi$. $\left(\kappa \mathrm{mp}^{\diamond}\right)$ is equivalent to the property $\left(\kappa \mathrm{cw}^{\diamond}\right)$ : If $\kappa^{w}(\varphi)<$ $\infty$ then $\kappa^{w}(w)=0$. This implies $(*)$ : when $\kappa^{w}\left([\varphi]^{F_{\mathfrak{R}}}\right)<\infty$, then $\max \left\{0, \kappa^{w}(w)-\right.$ $\left.\kappa^{w}\left([\varphi]^{F_{\mathfrak{R}}}\right)\right\}=0$ ! Thus, for a model $\mathfrak{R}$ with this property $\vDash$ and $\vDash^{*}$ agree: Let us verify this for $\varphi>\psi$

$$
\begin{aligned}
& w \vDash_{\mathfrak{R}} \varphi>\psi \\
& \text { iff } \left.\quad \kappa^{w}\left([\varphi]^{F_{\mathfrak{R}}}\right)=\infty \text { or }\left(\kappa_{[\varphi]^{\vDash_{\mathfrak{R}}}}^{w}\left([\neg \psi]^{\vDash_{\mathfrak{R}}}\right)\right)>0 \text { and } \kappa^{w}\left([\varphi]^{\vDash_{\mathfrak{R}}}\right)<\infty\right) \quad \text { (def. } \vDash_{\mathfrak{R}} \text { ) } \\
& \text { iff } \kappa^{w}\left([\varphi]^{\vDash_{\mathfrak{R}}}\right)=\infty \text { or } \kappa_{[\varphi]^{w}{ }_{\mathfrak{R}}}^{{ }^{\natural}}\left([\neg \psi]^{\vDash_{\mathfrak{R}}}\right)>\max \left\{0, \kappa^{w}(w)-\kappa^{w}\left([\varphi]^{\vDash_{\mathfrak{R}}}\right\}\right. \\
& \text { iff } \kappa^{w}\left([\varphi]^{F_{\mathfrak{R}}^{*}}\right)=\infty \text { or } \kappa_{[\varphi]^{w}}^{F_{\mathfrak{R}}^{*}}\left([\neg \psi]^{F_{\mathfrak{R}}^{*}}\right)>\max \left\{0, \kappa^{w}(w)-\kappa^{w}\left([\varphi]^{F_{\mathfrak{R}}^{*}}\right)\right\} \\
& \text { iff } \quad w \vDash_{\mathfrak{R}}^{*} \varphi>\psi
\end{aligned}
$$

But then $w \nvdash_{\mathfrak{R}}^{*} \varphi$. Thus $\nvdash^{*} \varphi$ in the alternative standard ranking semantics.

§8. Acknowledgments. I thank Franz Huber for stimulating discussions on his versions of the ranking semantics, Niels Skovgaard-Olsen for extensive discussions on initial ideas, and Wolfgang Spohn, participants of the Logica 2017, and members of the 'What-if?' research group for helpful comments on an earlier version. This research was funded by the Deutsche Forschungsgemeinschaft (DFG) Research Unit FOR 1614. 


\section{BIBLIOGRAPHY}

Alchourrón, C. E., Gärdenfors, P., \& Makinson, D. (1985). On the logic of theory change: Partial meet contraction and revision functions. Journal of Symbolic Logic, 50, 510-530.

Berto, F., French, R., Priest, G., \& Ripley, D. (2018). Williamson on counterpossibles. Journal of Philosophical Logic, 47(4), 693-713.

Burgess, J. (1981). Quick completeness proofs for some logics of conditionals. Notre Dame Journal of Formal Logic, 22, 76-84.

Bjerring, J. C. (2014). On counterpossibles. Philosophical Studies, 168, 327-353.

Brogaard, B. \& Salerno, J. (2013). Remarks on counterpossibles. Synthese, 190, 639-660.

Chellas, B. F. (1975). Basic conditional logic. Journal of Philosophical Logic, 4, 133-153.

Darwiche, A. \& Pearl, J. (1997). On the logic of iterated belief revision. Artificial Intelligence, 89(1-2), 1-29.

Fine, K. (1975). Review of counterfactuals. Mind, 84, 451-458.

Friedman, N. \& Halpern, J. (2001). Plausibility measures and default reasoning. Journal of the Association for Computing Machinery, 48(4), 648-685.

Gärdenfors, P. (1982). Imaging and conditionalization. The Journal of Philosophy, 79(12), 747-760.

Goldszmidt, M. \& Pearl, J. (1996). Qualitative probabilities for default reasoning, belief revision, and causal modelling. Artificial Intelligence, 84, 57-112.

Halpern, J. (2003). Reasoning About Uncertainty. Cambridge, MA: MIT Press.

Huber, F. (2006). Ranking functions and rankings on languages. Artificial Intelligence, 170, 462-471.

Huber, F. (2007a). The logic of theory assessment. Journal of Philosophical Logic, 36, 511-538.

Huber, F. (2007b). The consistency argument for ranking functions. Studia Logica, 86(2), 299-329.

Huber, F. (2014). New foundations for counterfactuals. Synthese, 91, 2167-2193.

Huber, F. (2015). What should I believe about what would have been the case? Journal of Philosophical Logic, 44, 81-110.

Huber, F. (2017). Why follow the royal rule? Synthese, 194(5), 1565-1590.

Kment, B. (2014). Modality and Explanatory Reasoning. Oxford: Oxford University Press.

Lauer, S. (2017). 'I believe' in a ranking-theoretic analysis of 'believe'. In van Gessel, T. and Roelofsen, F., editors. Proceedings of the $21^{\text {st }}$ Amsterdam Colloquium. Amsterdam: ILLC, pp. 335-344.

Lewis, D. (1971). Completeness and decidability of three logics of counterfactual conditionals. Theoria, 37(1), 74-85.

Lewis, D. (1973). Counterfactuals and comparative possibility. Journal of Philosophical Logic, 2, 418-446.

Loewer, B. M. (1979). Cotenability and counterfactual logics. Journal of Philosophical Logic, 8(1), 99-115.

Mares, E. D. \& Fuhrmann, A. (1995). A relevant theory of conditionals. Journal of Philosophical Logic, 24, 645-665.

Mayer, J. C. (1981). A misplaced thesis of conditional logic. Journal of Philosophical Logic, 10(2), 235-238.

Nolan, D. (1997). Impossible worlds: A modest approach. Notre Dame Journal of Formal Logic, 38, 535-572.

Raidl, E. (2018). Ranking semantics for doxastic necessities and conditionals. In Pavel, A. \& Lávička, T., editors. Logica Yearbook 2017. London: College Publications, pp. 223-238. 
Raidl, E. \& Skovgaard-Olsen, N. (2017). Simulating Lewis/Stalnaker Conditionals in Ranking Theory. Unpublished manuscript.

Spohn, W. (1988). Ordinal conditional functions. A dynamic theory of epistemic states. In Harper, W. L. and Skyrms, B. editors. Causation in Decision, Belief Change, and Statistics, Vol. 2. Dordrecht: Kluwer, pp. 105-134.

Spohn, W. (2012). The Laws of Belief: Ranking Theory and its Philosophical Applications. Oxford: Oxford University Press.

Spohn, W. (2015). Conditionals: A unifying ranking-theoretic perspective. Philosopher's Imprint, 15(1), 1-30.

Unterhuber, M. (2016). Beyond system P - Hilbert-Style convergence results for conditional logics with a connexive twist. IFCoLog Journal of Logics and their Application, 3(3), 376-412.

Williamson, T. (2007). The philosophy of philosophy. Oxford: Oxford University Press.

Williamson, T. (2018). Counterpossibles. Topoi, 37(3), 357-368.

\author{
DEPARTMENT OF PHILOSOPHY \\ UNIVERSITY OF KONSTANZ \\ POSTFACH D6, 78457 KONSTANZ, GERMANY \\ E-mail: eric.3.raidl@uni-konstanz.de
}

